Pacific Journal of Mathematics

SOMORPHISMS MODULO THE COMPACT OPERATORS OF 


\title{
ISOMORPHISMS MODULO THE COMPACT OPERATORS OF NEST ALGEBRAS
}

\author{
Constantin Apostol ANd Frank GilfeAther
}

\begin{abstract}
Let $\mathscr{A}$ and $\mathscr{B}$ be nest algebras of operators on a Hilbert space with finite-rank nest projections $\mathscr{N}_{\mathscr{A}}=\left\{P^{(n)}\right\}$ and $\mathscr{N}_{\mathscr{B}}=\left\{Q^{(n)}\right\}, n \in \mathbf{N}$, respectively. Let $P_{n}=P^{(n+1)}-P^{(n)}$ and $Q_{n}=Q^{(n+1)}-Q^{(n)}$ be the block diagonal projections for the two nests. $\mathscr{A}$ and $\mathscr{B}$ are thus the upper triangular matrices with respect to the decompositions determined by $\left\{P_{n}\right\}_{n \in \mathrm{N}}$ and $\left\{Q_{n}\right\}_{n \in \mathrm{N}}$ respectively. It is easy to see that $\mathscr{A}$ is isomorphic to $\mathscr{B}$ if and only if rank $P_{n}=$ rank $Q_{n}$ for all $n$. J. Plastiras has shown that the quasitriangular algebra $\mathscr{A}+K(H)$, that is $\mathscr{A}$ plus the compact operators, is isomorphic to $\mathscr{B}+K(H)$ if and only if there exist integers $n_{0}$ and $m_{0}$ so that rank $P^{\left(n_{0}+n\right)}=\operatorname{rank} Q^{\left(m_{0}+n\right)}$ for all $n$. Using different techniques this paper shows that the image of $\mathscr{A}$ in the Calkin algebra $\tilde{\mathscr{A}}$ is isomorphic to $\tilde{\mathscr{B}}$ if and only if there exist integers $n_{0}$ and $m_{0}$ so that rank $P_{n_{0}+n}=\operatorname{rank} Q_{m_{0}+n}$ for all $n$.
\end{abstract}

Nest algebras were introduced by $\mathbf{J}$. Ringrose as generalizations of reflexive triangular operator algebras [6]. Recently there has been a spate of important and deep developements in the theory of nest and the related quasitriangular operator algebras. A discussion of these recent results can be found in W. Arveson's CBMS conference lecture notes [1] while earlier and related work is aptly described in a survey by $\mathrm{J}$. Erdos [2]. We note that for nest algebras one has that $\mathscr{A}+K(H)$ is norm closed and $\tilde{\mathscr{A}}=[A+K(H)] / K(H)=\mathscr{A} /[\mathscr{A} \cap K(H)]$ so that our results concern as well the isomorphism classes of certain quasitriangular algebras modulo the compact operators [3].

We illustrate the obvious different results concerning the isomorphisms of these different algebras with some simple examples. Let $\mathscr{A}\left(n_{1}, n_{2}, \ldots\right)$ represent the nest algebra with block diagonal projections having ranks $\left\{n_{i}\right\}_{i \in N^{*}}$. Clearly $\mathscr{A}(2,1,1, \ldots)$ is not isomorphic to $\mathscr{A}(1,2,1,1 \ldots)$ however the corresponding quasitriangular algebras are isomorphic and hence are isomorphic modulo the compact operators. Moreover Plastiras' result [4] shows $\mathscr{A}(1,2,2, \ldots)+K(H)$ is not isomorphic to $\mathscr{A}(2,2, \ldots)+K(H)$, while the result in this paper shows $\tilde{\mathscr{A}}(1,2,2, \ldots)$ is isomorphic to $\tilde{\mathscr{A}}(2,2, \ldots)$. Finally our result shows that $\tilde{\mathscr{A}}(1,2,1,2, \ldots)$ is not isomorphic to $\tilde{\mathscr{A}}(1,1,2,1,1,2, \ldots)$. This result can be restated in terms of the nest projections as: The algebras $\tilde{\mathscr{A}}$ and $\tilde{\mathscr{B}}$ are 
isomorphic if and only if there exists integers $n_{0}, m_{0}, k_{0}$ so that the nest projections satisfy $\operatorname{rank} P^{\left(n_{0}+n\right)}=k_{0}+\operatorname{rank} Q^{\left(m_{0}+n\right)}$ for all $n$. For Pastiras' result about quasitriangular algebras one has $k_{0}=0$.

We take this opportunity to express our gratitude to W. Arveson, K. Davidson and D. Larson for discussions on the subject. The second author also wishes to acknowledge partial support from the SERC (British) and the National Bureau of Standards and both authors acknowledge support from the National Science Foundation.

1. Preliminaries. Throughout this paper $H$ will denote a complex infinite dimensional, separable Hilbert space, $L(H)$ will denote the algebra of all bounded linear operators acting in $H$ and $K(H)$ the ideal in $L(H)$ of compact operators. For every $T \in L(H)$, the symbol $\tilde{T}$ will denote the image of $T$ in the Calkin algebra $L(H) / K(H)$ and for every $M \in L(H)$ we shall put $\tilde{M}=\{\tilde{T}: T \in M\}$.

Let $\mathscr{A}, \mathscr{B}$ be nest algebras of operators in $H$, with the finite-rank nest projections $\left\{P^{(n)}\right\}_{n \in \mathrm{N}},\left\{Q^{(n)}\right\}_{n \in \mathrm{N}}$. Correspondingly we consider the block-diagonal projections $\left\{P_{n}\right\}_{n \in \mathbf{N}},\left\{Q_{n}\right\}_{n \in \mathbf{N}}$, defined by

$$
\begin{gathered}
P_{1}=P^{(1)}, \quad Q_{1}=Q^{(1)}, \quad P_{n+1}=P^{(n+1)}-P^{(n)}, \\
Q_{n+1}=Q^{(n+1)}-Q^{(n)}, \quad n \in \mathbf{N} .
\end{gathered}
$$

For each $k \geq 0$ define the operators

$$
D_{k}: L(H) \rightarrow L(H), \quad \Delta_{k}: L(H) \rightarrow L(H)
$$

by the equations

$$
D_{k} T=\sum_{n \in \mathbf{N}} P_{n} T P_{n+k}, \quad \Delta_{k} T=\sum_{n \in \mathbf{N}} Q_{n} T Q_{n+k}, \quad T \in L(H) .
$$

It is plain that both $D_{k}$ and $\Delta_{k}$ are norm-one projections that leave invariant $K(H)$. The corresponding quotient operators $\tilde{D}_{k}, \tilde{\Delta}_{k}$ are normone projections, too. Observe that we have $D_{k} \mathscr{A} \subset \mathscr{A}$ and $\Delta_{k} \mathscr{B} \subset \mathscr{B}$. For each $n \in \mathbf{N}$ put

$$
\mathscr{A}_{(n)}=\left\{A \in \mathscr{A}: D_{k} \mathscr{A}=0,0 \leq k<n\right\} .
$$

The core of the nest algebra $\mathscr{A}$ denoted by $\mathscr{A}_{0}$, is the commutant of $\mathscr{A} \cap \mathscr{A}^{*}$. Clearly $\mathscr{A}_{0}$ is an abelian von Neumann algebra and we define

$$
\mathscr{A}_{(0)}=\mathscr{A}_{0}+\mathscr{A}_{(1)} \text {. }
$$

Then $\mathscr{A}_{(0)}$ is a closed subalgebra in $\mathscr{A}$ and $\mathscr{A}_{(n)}$, for $n \geq 1$, is an ideal in both $\mathscr{A}_{\tilde{D}}$ and $\mathscr{A}_{(0)}$. Since $\tilde{\mathscr{A}}$ is norm-closed and we have $\tilde{\mathscr{A}}_{(n)}=\tilde{\mathscr{A}} \cap$ $\left(\cap_{k=0}^{n-1} \operatorname{ker} \tilde{D}_{k}\right), n \in \mathbf{N}$, we deduce that $\tilde{\mathscr{A}}_{(n)}(n \geq 1)$ is closed. The algebra 
$\tilde{\mathscr{A}}$ is the direct topological sum $\tilde{\mathscr{A}}=\left(\tilde{D}_{0} \tilde{\mathscr{A}}\right)+\tilde{\mathscr{A}}_{(1)}$ and an elementary argument shows that $\tilde{\mathscr{A}}_{0}$ is closed and in particular $\tilde{\mathscr{A}}_{(0)}=\tilde{\mathscr{A}}_{0}+\tilde{\mathscr{A}}_{(1)}$ is closed. We shall use analogous notation involving $\mathscr{B}$.

The first two lemmas below express well-known basic facts in the theory of nest algebras. We sketch their proofs for the sake of completeness. The proof of the first we learned from W. Arveson and the second lemma is due to D. Larson.

1.1. Lemma. Let $\mathscr{M}=\left\{T \in L(H):\left(1-P^{(n)}\right) T Q^{(n)}=0, n \in \mathbf{N}\right\}$. Then we have

$$
\mathscr{M}+K(H)=\left\{T \in L(H): \varlimsup_{n \rightarrow \infty}\left\|\left(1-P^{(n)}\right) T Q^{(n)}\right\|=0\right\}
$$

and consequently $\tilde{\mathscr{M}}$ is closed.

Proof. Let $\mathscr{A}_{1}$ be the nest algebra in $L(H \oplus H)$ determined by the nest $\left\{P^{(n)} \oplus Q^{(n)}\right\}_{n \in \mathbf{N}}$. It is easy to see that we have

$$
\left(\begin{array}{ll}
0 & T \\
0 & 0
\end{array}\right) \in \mathscr{A}_{1}+K(H \oplus H) \quad \text { iff } \quad \varlimsup_{n \rightarrow \infty}\left\|\left(1-P^{(n)}\right) T Q^{(n)}\right\|=0 .
$$

Hence if $\varlimsup_{n \rightarrow \infty}\left\|\left(1-P^{(n)}\right) T Q^{(n)}\right\|=0$ we, can find $K_{l, J} \in K(H)$ such that

$$
\left(\begin{array}{cc}
K_{1,1} & T+K_{1,2} \\
K_{2,1} & K_{2,2}
\end{array}\right) \in \mathscr{A}_{1} .
$$

Since $\left\{P^{(n)} \oplus Q^{(n)}\right\}_{n \in \mathbf{N}}$ are invariant projections for $\mathscr{A}_{1}$ we easily deduce that $T+K_{1,2} \in \mathscr{M}$ and in particular

$$
\mathscr{M}+K(H) \supset\left\{T \in L(H): \varlimsup_{n \rightarrow \infty}\left\|\left(1-P^{(n)}\right) T Q^{(n)}\right\|=0\right\} .
$$

The opposite inclusion is obvious.

1.2. Lemma. Let $\mathscr{P} \subset \mathscr{A}$ be a finite lattice of commuting projections. Then there exists $A \in \mathscr{A}$, invertible in $\mathscr{A}$ such that $A^{-1} P A$ is self-adjoint for every $P \in \mathscr{P}$.

Proof. Let $T \in L(H)$ be any invertible operator such that $T^{-1} P T$, $P \in \mathscr{P}$ is self-adjoint. If we put $\mathscr{C}=T^{-1} \mathscr{A} T$, then by the nature of our nest we can find a unitary operator $U \in L(H)$ such that $\mathscr{A}=U * \mathscr{C} U$. Since obviously $A=T U$ and $A^{-1}$ belong to $\mathscr{A}$ and $A^{-1} P A \in \mathscr{P}$ is self-adjoint, the proof is concluded. 
1.3. Lemma. Let $\tilde{\mathscr{P}} \subset \tilde{\mathscr{A}}$ be a finite lattice of commuting idempotents. Then there exists or finite lattice of commuting projections $\mathscr{P}_{0} \subset \mathscr{A}$ such that the map

$$
P \rightarrow \tilde{P} \in \tilde{\mathscr{P}}, \quad P \in \mathscr{P}_{0}
$$

is a lattice isomorphism. Moreover, if $\tilde{\mathscr{P}} \subset \tilde{\mathscr{A}}_{(0)}$ we may suppose $\mathscr{P}_{0} \subset \mathscr{A}_{(0)}$.

Proof. We can choose $\left\{\tilde{A}_{n}\right\}_{n=1}^{k} \subset \tilde{\mathscr{P}}$ such that $\tilde{A}_{n} \tilde{A}_{m}=0, n \neq m$ and $\left\{\tilde{A}_{n}\right\}_{n=1}^{k}$ generates $\tilde{\mathscr{P}}$. Pick $A_{n}^{\prime} \in \mathscr{A}\left(\right.$ or $\left.A_{n}^{\prime} \in \mathscr{A}_{(0)}\right)$ such that $\tilde{A}_{n}^{\prime}=\tilde{A}_{n}$. Since $\sigma\left(\tilde{A}_{1}^{\prime}\right) \subset\{0,1\}, \sigma\left(A_{1}^{\prime}\right)$ can accumulate only at 0 or 1 . Let $\Gamma \subset \rho\left(A_{1}^{\prime}\right)$ (the resolvent set of $A_{1}^{\prime}$ ) be a simple admissible contour surrounding 1 and leaving 0 in its exterior. Because $\rho\left(A_{1}^{\prime}\right)$ is connected we have $\left(\lambda-A_{1}^{\prime}\right)^{-1}$ $\in \mathscr{A}$ (or $\left.\in \mathscr{A}_{(0)}\right)$ for $\lambda \in \rho\left(A_{1}^{\prime}\right)$, thus if we put

$$
P_{1}=\frac{1}{2 \pi i} \int_{\Gamma}\left(\lambda-A_{1}^{\prime}\right)^{-1} d \lambda,
$$

we have $P_{1}=P_{1}^{2} \in \mathscr{A}$ (or $\in \mathscr{A}_{(0)}$ ) and $\tilde{P}_{1}=\tilde{A}_{1}$. Further we may suppose $P_{1} A_{k}^{\prime}=A_{k}^{\prime} P_{1}=0,2 \leq k \leq n$ (after possibly a compact perturbation) and then produce $P_{2}$ as before. In a finite number of steps we can produce $\left\{P_{n}\right\}_{n=1}^{k} \subset \mathscr{A}\left(\right.$ or $\left.\mathscr{A}_{(0)}\right)$ such that $\mathscr{P}_{0}$, the lattice generated by $\left\{P_{n}\right\}_{n=1}^{k}$ has the desired properties.

\subsection{Lemma. Let $\tilde{A}=\tilde{A}^{2} \in \tilde{\mathscr{A}}$. Then $\tilde{D}_{0} \tilde{A}=0$ iff $\tilde{A}=0$.}

Proof. Using Lemma 1.3 we can find $P=P^{2} \in \mathscr{A}$ such that $\tilde{P}=\tilde{A}$. By Lemma 1.2 , we also can find $A_{0} \in \mathscr{A}$, invertible in $\mathscr{A}$, such that $A_{0}^{-1} P A_{0}$ is selfadjoint. Since we obviously have

$$
A_{0}^{-1} P A_{0}=D_{0}\left(A_{0}^{-1} P A_{0}\right)=\left(D_{0} A_{0}^{-1}\right)\left(D_{0} P\right)\left(D_{0} A_{0}\right)
$$

the equivalence in the statement follows.

1.5. LemMA. Let $f, g: \mathbf{N} \rightarrow \mathbf{N}$ be strictly monotone functions and let $T \in L(H), \varepsilon>0$. Then there exists an infinite set $\sigma \subset \mathbf{N}$ such that

$$
K=\sum_{n \in \sigma} P_{f(n)} T Q_{g(n)}-\left(\sum_{n \in \sigma} P_{f(n)}\right) T\left(\sum_{n \in \sigma} Q_{g(n)}\right) \in K(H), \quad\|K\|<\varepsilon .
$$

Proof. We shall determine by induction a sequence $\left\{r_{k}\right\}_{k \in \mathbf{N}}$ such that

$$
r_{1}=1, \quad\left\|P_{f\left(r_{k}\right)} T Q_{g\left(r_{m}\right)}\right\| \leq \frac{\varepsilon}{2^{k+m}}, \quad k \neq m .
$$

Observe that if $\left\{r_{k}\right\}_{k=1}^{m}$ is determined we have

$$
\lim _{n \rightarrow \infty}\left\|P_{f(n)} T Q_{g\left(n_{k}\right)}\right\|=\lim _{n \rightarrow \infty}\left\|P_{f\left(r_{k}\right)} T Q_{g(n)}\right\|=0,
$$


thus we can choose $r_{m+1}$, large enough. If we put $\sigma=\left\{r_{k}\right\}_{k \in N}$, then

$$
\|K\| \leq \sum_{k \neq m}\left\|P_{f\left(r_{k}\right)} T Q_{g\left(r_{m}\right)}\right\| \leq \varepsilon \sum_{k \neq m} 2^{-(k+m)}<\varepsilon
$$

and because $P_{f\left(r_{k}\right)} T P_{g\left(r_{m}\right)}$ is finite-rank, $k$ is thus compact.

2. Isomorphisms. In the sequel $\alpha$ will denote a fixed isomorphism

$$
\alpha: \tilde{\mathscr{A}} \rightarrow \tilde{\mathscr{B}} \text {. }
$$

Let $\mathscr{N}$ denote the complemented lattice of all subsets in $\mathbf{N}$ and put

$$
\mathscr{N}_{0}=\{\sigma \in N: \sigma \text { is finite }\} \text {. }
$$

We define an equivalence relation in $\mathscr{N}$ by

$$
\sigma \simeq \delta \quad \text { iff } \quad(\sigma \backslash \delta) \cup(\delta \backslash \sigma) \in \mathscr{N}_{0} .
$$

The resulting set of equivalence classes in $\mathscr{N}$ will be denoted by $\hat{\mathscr{N}}$, i.e. if $\sigma \in \mathbf{N}$ we put

$$
\hat{\sigma}=\{\delta \in \mathscr{N}: \delta \simeq \sigma\} .
$$

For every $\sigma \in \mathscr{N}_{0}$ we have $\hat{\sigma}=\hat{\phi}$ where $\phi$ is the empty set. For every $\sigma$, $\delta \in \mathscr{N}$, the lattice operations

$$
\hat{\sigma} \cup \hat{\delta}=\widehat{\sigma \cup \delta} \text { and } \hat{\sigma} \cap \hat{\delta}=\overline{\sigma \cap \delta}
$$

are well defined and thus $\hat{\mathscr{N}}$ becomes a complemented lattice.

Let $\tau: \mathscr{N} \rightarrow \mathscr{N}$ be the set-translation map defined for $\sigma \in \mathscr{N}$ by

$$
\tau(\sigma)=\{n+1: n \in \sigma\} .
$$

We also define $\tau^{*}: \mathscr{N} \rightarrow \mathscr{N}$ by

$$
\tau^{*}(\sigma)=\{n: n+1 \in \sigma\}, \quad \sigma \in \mathscr{N} .
$$

The map $\tau^{*}$ is not injective because $\tau^{*}(\varnothing)=\tau^{*}(\{1\})=\varnothing$. However it clearly follows that $\tau^{*} \tau(\sigma)=\sigma$ for $\sigma \in \mathcal{N}$ and $\tau \tau^{*}(\sigma)=\sigma, \sigma \in \tau(\mathscr{N})$.

Defining $\hat{\tau}: \hat{\mathscr{N}} \rightarrow \hat{\mathscr{N}}$ and $\hat{\tau}^{*}: \hat{\mathscr{N}} \rightarrow \hat{\mathscr{N}}$ by

$$
\hat{\tau}(\hat{\boldsymbol{\sigma}})=\widehat{\tau(\boldsymbol{\sigma})} \text { and } \hat{\tau}^{*}(\hat{\boldsymbol{\sigma}})=\widehat{\tau^{*}(\boldsymbol{\sigma})}
$$

we have $\hat{\tau} \hat{\tau}^{*}(\hat{\sigma})=\hat{\tau}^{*} \hat{\tau}(\hat{\sigma})=\hat{\sigma}$ and thus $\hat{\tau}$ is invertible and $\hat{\tau}^{-1}=\hat{\tau}^{*}$.

Let $P=P^{2} \in \mathscr{A}$ and put

$$
\operatorname{supp}_{\mathscr{A}}(P)=\left\{n \in \mathbf{N}: P_{n} P P_{n} \neq 0\right\} .
$$

By Lemma 1.2 we know that $P_{n} P P_{n}$ is a projection, thus if $P^{\prime}=P^{\prime 2} \in A$ and $P-P^{\prime} \in K(H)$, we have $D_{0}\left(P-P^{\prime}\right) \in K(H)$ and this obviously implies $\operatorname{supp}_{\mathscr{A}}(P) \simeq \operatorname{supp}_{\mathscr{A}}\left(P^{\prime}\right)$. 
Let $\tilde{A}=\tilde{A}^{2} \in \tilde{\mathscr{A}}$. The above observation allows us to define $\operatorname{supp}_{A}(\tilde{A}) \in \hat{\mathscr{N}}$ by

$$
\left.\operatorname{supp}_{\mathscr{A}}(\tilde{A})=\widehat{\operatorname{supp}_{\mathscr{A}}(P}\right) \text {, }
$$

where $P \in \mathscr{A}$ and $\tilde{P}=\tilde{\mathscr{A}}$ is produced by Lemma 1.3. For each $\sigma \in \mathscr{N}$ we define

$$
P_{\sigma}=\sum_{n \in \sigma} P_{n} \quad \text { if } \sigma \neq \varnothing \text { and } P_{\varnothing}=0 .
$$

Because $\alpha\left(\tilde{P}_{\sigma}\right)$ is an idempotent in $\tilde{\mathscr{B}}$ we can define $\hat{\alpha}: \hat{\mathscr{N}} \rightarrow \hat{\mathscr{N}}$, by

$$
\hat{\alpha}(\hat{\sigma})=\operatorname{supp}_{\mathscr{B}}\left(\alpha\left(\tilde{P}_{\sigma}\right)\right) .
$$

Our main objective in this section will be to obtain some basic properties of the isomorphism $\alpha$ and of the map $\hat{\alpha}$. However, we shall need as an auxiliary tool a result on isomorphisms between $\tilde{\mathscr{A}}_{(0)}$ and $\tilde{\mathscr{B}}_{(0)}$.

2.1. Lemma. For every isomorphism $\psi: \tilde{\mathscr{A}}_{(0)} \rightarrow \tilde{\mathscr{B}}_{(0)}$, the compression

$$
\tilde{\Delta}_{0} \psi ; \tilde{\mathscr{A}}_{0} \rightarrow \tilde{\mathscr{B}}_{0}
$$

is an isomorphism, moreover

$$
\left(\tilde{\Delta}_{0} \psi \mid \tilde{\mathscr{A}}_{0}\right)^{-1}=\tilde{D}_{0} \psi^{-1} \mid \tilde{\mathscr{B}}_{0} \quad \text { and } \quad \psi\left(\tilde{\mathscr{A}}_{(1)}\right)=\tilde{\mathscr{B}}_{(1)} .
$$

Proof. Let $\tilde{A}_{0} \in \tilde{\mathscr{A}}_{0}$ be such that $\tilde{\Delta}_{0} \psi\left(\tilde{A}_{0}\right)=0$ and let $P=P^{2} \in \mathscr{A}_{0}$ be such that $\tilde{A}_{0}^{*} \tilde{A}_{0} \tilde{P}=\left\|\tilde{A}_{0}\right\|^{2} \tilde{P}, \tilde{P} \neq 0$. Since obviously $\tilde{\Delta}_{0} \psi \mid \tilde{\mathscr{A}}_{0}$ is an homomorphism, we have

$$
\left\|\tilde{A}_{0}\right\|^{2} \tilde{\Delta}_{0} \psi(\tilde{P})=\tilde{\Delta}_{0} \psi\left(\tilde{A}_{0}^{*} \tilde{A_{0}} \tilde{P}\right)=\left(\tilde{\Delta}_{0} \psi\left(\tilde{A}_{0}^{*}\right)\right)\left(\tilde{\Delta}_{0} \psi\left(\tilde{A}_{0}\right)\right)\left(\tilde{\Delta}_{0} \psi(\tilde{P})\right)=0
$$

and by Lemma 1.4, $\tilde{\Delta}_{0} \psi(\tilde{P}) \neq 0$, thus we derive $\tilde{A}_{0}=0$. This shows that $\tilde{\Delta}_{0} \psi \mid \tilde{\mathscr{A}}_{0}$ and $\tilde{D}_{0} \psi^{-1} \mid \tilde{\mathscr{B}}_{0}$ are injective. Now let $A_{1} \in \mathscr{A}_{(1)}$ and $B_{1} \in \psi\left(\tilde{A}_{1}\right)$. If $\tilde{B}_{1} \notin \tilde{\mathscr{B}}_{(1)}$ we can find $\gamma>0$ and a strictly monotone function $f$ : $\mathbf{N} \rightarrow \mathbf{N}$ such that $\left\|Q_{f(n)} B_{1} Q_{f(n)}\right\| \geq \gamma$, for all $n \in N$. Applying Lemma 1.5 we can find $\sigma \in \mathcal{N} \backslash \mathscr{N}_{0}$ such that

$$
Q_{f(\sigma)} B_{1} Q_{f(\sigma)}-\Delta_{0}\left(Q_{f(\sigma)} B_{1} Q_{f(\sigma)}\right) \in K(H), \quad Q_{f(\sigma)} B_{1} Q_{f(\sigma)} \notin K(H) .
$$

Since $\tilde{\mathscr{A}}_{(1)}$ is an ideal in $\tilde{\mathscr{A}}_{(0)}$, we have

$$
\psi^{-1}\left(\tilde{Q}_{f(\sigma)} \tilde{B}_{1} \tilde{Q}_{f(\sigma)}\right)=\psi^{-1}\left(\tilde{Q}_{f(\sigma)}\right) \tilde{A}_{1} \psi^{-1}\left(\tilde{Q}_{f(\sigma)}\right) \in \tilde{\mathscr{A}}_{(1)},
$$

and thus $\tilde{D}_{0} \psi^{-1}\left(\tilde{Q}_{f(\sigma)} \tilde{B}_{1} \tilde{Q}_{f(\sigma)}\right)=0$, contradicting the injectivity of $\tilde{D}_{0} \psi^{-1} \mid \tilde{\mathscr{B}}_{0}$. The conclusion is that we have $\psi\left(\tilde{\mathscr{A}}_{(1)}\right) \subset \tilde{\mathscr{B}}_{(1)}$ and by symmetry $\psi^{-1}\left(\tilde{\mathscr{B}}_{(1)}\right) \subset \tilde{\mathscr{A}}_{(1)}$. Finally using the relation $\psi\left(\tilde{\mathscr{A}}_{(1)}\right)=\tilde{\mathscr{B}}_{(1)}$, we infer 
that

$\left(\tilde{D}_{0} \psi^{-1}\right)\left(\tilde{\Delta}_{0} \psi\left(\tilde{A}_{0}\right)\right)=\left(\tilde{D}_{0} \psi^{-1}\right)\left(\psi\left(\tilde{A}_{0}\right)\right)=\tilde{A}_{0}, \quad$ whenever $\tilde{A}_{0} \in \tilde{\mathscr{A}}_{0}$ and

$\left(\tilde{\Delta}_{0} \psi\right)\left(\tilde{D}_{0} \psi^{-1}\left(\tilde{B}_{0}\right)\right)=\left(\Delta_{0} \psi\right)\left(\psi^{-1}\left(\tilde{B}_{0}\right)\right)=\tilde{B}_{0} \quad$ whenever $\tilde{B}_{0} \in \tilde{\mathscr{B}}_{0}$.

2.2. ThEOREM. The compression $\tilde{\Delta}_{0} \alpha: \tilde{D}_{0} \tilde{\mathscr{A}} \rightarrow \tilde{\Delta}_{0} \tilde{\mathscr{B}}$ is an isomorphism, moreover

$$
\left(\tilde{\Delta}_{0} \alpha \mid \tilde{D}_{0} \tilde{\mathscr{A}}\right)^{-1}=\tilde{D}_{0} \alpha^{-1} \mid \tilde{\Delta}_{0} \tilde{\mathscr{B}}, \alpha\left(\tilde{\mathscr{A}}_{(1)}\right)=\tilde{\mathscr{B}}_{(1)} \quad \text { and } \quad \alpha\left(\tilde{\mathscr{A}}_{(0)}\right)=\tilde{\mathscr{B}}_{(0)} .
$$

Proof. The properties of $\tilde{\Delta}_{0} \alpha \mid \tilde{D}_{0} \tilde{\mathscr{A}}$ and the relation $\alpha\left(\tilde{\mathscr{A}}_{(1)}\right)=\tilde{\mathscr{B}}_{(1)}$, can be derived as in the proof of Lemma 2.1, choosing $P \in D_{0} \mathscr{A}$ (in place of $\left.P \in \mathscr{A}_{0}\right)$. Let $A \in \mathscr{A}_{(0)}$ and $B \in \alpha(\tilde{A})$. For every $\tilde{T} \in \tilde{\mathscr{A}}$ we have

$\tilde{A} \tilde{T}-\tilde{T} \tilde{A} \in \tilde{\mathscr{A}}_{(1)} \quad$ and $\quad \tilde{B} \alpha(\tilde{T})-\alpha(\tilde{T}) \tilde{B}=\alpha(\tilde{A} \tilde{T}-\tilde{T} \tilde{A}) \in \tilde{\mathscr{B}}_{(1)}$, consequently for $\tilde{S} \in \tilde{\mathscr{B}}$

$$
\tilde{B} \tilde{S}-\tilde{S} \tilde{B} \in \tilde{\mathscr{B}}_{(1)} \quad \text { and } \quad\left(\tilde{\Delta}_{0} \tilde{B}\right)\left(\tilde{\Delta}_{0} \tilde{S}\right)-\left(\tilde{\Delta}_{0} \tilde{S}\right)\left(\Delta_{0} \tilde{B}\right)=0 .
$$

In particular $\tilde{\Delta}_{0} \tilde{B}$ commutes with every $\tilde{B}_{0} \in \tilde{\Delta}_{0} \tilde{\mathscr{B}}$ and this implies $\tilde{\Delta}_{0} \tilde{B} \in \tilde{\mathscr{B}}_{0}$, or equivalently $\tilde{B} \in \tilde{\mathscr{B}}_{(0)}$. Thus we have $\alpha\left(\tilde{\mathscr{A}}_{(0)}\right) \subset \tilde{\mathscr{B}}_{(0)}$ and by symmetry $\alpha^{-1}\left(\tilde{\mathscr{B}}_{(0)}\right) \subset \tilde{\mathscr{A}}_{(0)}$.

2.3. Lemma. Let $P=P^{2} \in \mathscr{A}$ be such that $\operatorname{rank} P_{n} P P_{n}=n_{0}$ for $n \in \operatorname{supp}_{\mathscr{A}}(P)$. Then there exists $Q=Q^{2} \in \mathscr{B}$ such that

$$
\tilde{Q}=\alpha(\tilde{P}), \quad \operatorname{rank} Q_{n} Q Q_{n}=n_{0} \quad \text { for } n \in \operatorname{supp}_{\mathscr{B}}(Q) .
$$

Proof. Let $Q^{\prime}=Q^{\prime 2} \in \mathscr{B}$ be produced by Lemma 1.3 , such that $\tilde{Q}^{\prime}=\alpha(\tilde{P})$ and put

$$
\sigma=\left\{n \in \operatorname{supp}_{B}\left(Q^{\prime}\right): \operatorname{rank} Q_{n} Q^{\prime} Q_{n}>n_{0}\right\}
$$

and

$$
\delta=\left\{n \in \operatorname{supp}_{B}\left(Q^{\prime}\right): \operatorname{rank} Q_{n} Q^{\prime} Q_{n}<n_{0}\right\} .
$$

If $\sigma \notin \mathscr{N}_{0}$ we can find $T \in \Delta_{0} \mathscr{B}$ such that

$$
\tilde{T}^{n_{0}+1}=0, \quad \tilde{T}^{n_{0}} \neq 0 \quad \text { and }\left(\Delta_{0} Q^{\prime}\right) T=T\left(\Delta_{0} Q^{\prime}\right)=T .
$$


Applying Theorem 2.2 we deduce that $\tilde{D}_{0} \alpha^{-1}(\tilde{T})$ is nilpotent with

$$
\begin{gathered}
\left(\tilde{D}_{0} \alpha^{-1}(\tilde{T})\right)^{n_{0}} \neq 0 \text { and } \\
\left(\tilde{D}_{0} \tilde{P}\right)\left(\tilde{D}_{0} \alpha^{-1}(\tilde{T})\right)=\left(\tilde{D}_{0} \alpha^{-1}(\tilde{T})\right)\left(\tilde{D}_{0} \tilde{P}\right)=\tilde{D}_{0} \alpha^{-1}(\tilde{T}) .
\end{gathered}
$$

Since $D_{0} P=\sum_{n \in \Lambda} P_{n}^{\prime}$ where $\Lambda=\operatorname{supp}_{\mathscr{A}}(P)$ and $\operatorname{rank} P_{n}^{\prime}=n_{0}$ if we choose $S \in \tilde{D}_{0} \alpha^{-1}(\tilde{T})$ we have

$$
S-S_{0} \in K(H) \quad \text { and } \quad S_{0}^{n_{0}} \notin K(H), \quad \text { where } S_{0}=\sum_{n \in \Lambda} P_{n}^{\prime} S P_{n}^{\prime} .
$$

But this is a contradiction because $S_{0}$ is similar to an orthogonal direct sum of operators acting in spaces whose dimensions do not exceed $n_{0}$ and such essential quasinilpotents are nilpotents of order at most $n_{0}$. The conclusion is that we have $\sigma \in \mathscr{N}_{0}$ and analogously $\delta \in \mathscr{N}_{0}$. Now putting $B=Q^{\prime}-\sum_{n \in \sigma \cup \delta} Q_{n} Q^{\prime} Q_{n}$ we can define

$$
Q=\frac{1}{2 \pi i} \int_{\Gamma}(\lambda-B)^{-1} d \lambda
$$

as in Lemma 1.3.

2.4. Lemma. Let $P, P^{\prime} \in \mathscr{A}$ and $Q, Q^{\prime} \in \mathscr{B}$ be idempotents such that $\operatorname{supp}_{\mathscr{A}}(\tilde{P})=\operatorname{supp}_{\mathscr{A}}\left(\tilde{P}^{\prime}\right)$ and $\tilde{Q}=\alpha(\tilde{P}), \quad \tilde{Q}^{\prime}=\alpha\left(\tilde{P}^{\prime}\right)$. Then we have $\operatorname{supp}_{\mathscr{B}}(\tilde{Q})=\operatorname{supp}_{\mathscr{B}}\left(\tilde{Q}^{\prime}\right)$.

Proof. Put $\sigma=\operatorname{supp}_{\mathscr{B}}(Q), \sigma^{\prime}=\operatorname{supp}_{\mathscr{B}}\left(Q^{\prime}\right)$. Using Theorem 2.2 we derive $\tilde{D}_{0} \alpha^{-1}\left(\tilde{Q}_{\sigma}\right)$ and $\tilde{D}_{0} \alpha^{-1}\left(\tilde{Q}_{\sigma^{\prime}}\right) \in \tilde{\mathscr{A}}_{0}$ and in addition $\left(\tilde{D}_{0} \tilde{P}\right)\left(\tilde{D}_{0} \alpha^{-1}\left(\tilde{Q}_{\sigma}\right)\right)=\tilde{D}_{0} \tilde{P}$ and $\left(\tilde{D}_{0} \tilde{P}^{\prime}\right)\left(\tilde{D}_{0} \alpha^{-1}\left(\tilde{Q}_{\sigma^{\prime}}\right)\right)=\tilde{D}_{0} \tilde{P}^{\prime}$. Since $\tilde{D}_{0} \alpha^{-1}\left(\tilde{Q}_{\sigma}\right)=\tilde{P}_{\delta}$ and $\tilde{D}_{0} \alpha^{-1}\left(\tilde{Q}_{\sigma^{\prime}}\right)=\tilde{P}_{\delta^{\prime}}$ for some $\delta$ and $\delta^{\prime} \in \mathcal{N}$ we derive $\left(\tilde{D}_{0} \tilde{P}\right) \tilde{P}_{\delta \cap \delta^{\prime}}=\tilde{D}_{0} P, \quad\left(\tilde{D}_{0} \tilde{P}^{\prime}\right) \tilde{P}_{\delta \cap \delta^{\prime}}=\tilde{D}_{0} \tilde{P}^{\prime}, \quad\left(\tilde{\Delta}_{0} \tilde{Q}\right) \tilde{Q}_{\sigma \cap \sigma^{\prime}}=\tilde{\Delta}_{0} \tilde{Q}$, and $\left(\tilde{\Delta}_{0} \tilde{Q}^{\prime}\right) \tilde{Q}_{\sigma} \cap \sigma^{\prime}=\Delta_{0} \tilde{Q}^{\prime}$. Consequently we must have $\hat{\sigma} \cap \hat{\sigma}^{\prime} \supset \hat{\sigma}$ and $\hat{\sigma} \cap$ $\hat{\sigma}^{\prime} \supset \hat{\sigma}^{\prime}$ so it follows that $\hat{\sigma}=\hat{\sigma}^{\prime}$.

\subsection{Proposition. $\hat{\alpha}$ is a lattice isomorphism.}

Proof. Let $\sigma$ and $\delta \in \mathscr{N}$. Since $P_{\sigma \cap \delta}=P_{\sigma} P_{\delta}$ and $\alpha\left(\tilde{P}_{\sigma \cap \delta}\right)=$ $\alpha\left(\tilde{P}_{\sigma}\right) \alpha\left(\tilde{P}_{\delta}\right)$, choosing $\sigma^{\prime} \in \hat{\alpha}(\hat{\sigma})$ and $\delta^{\prime} \in \hat{\alpha}(\hat{\delta})$ and applying Theorem 2.2 we have $\tilde{Q}_{\sigma^{\prime}}=\tilde{\Delta}_{0} \alpha\left(\tilde{P}_{\sigma}\right), \tilde{Q}_{\delta^{\prime}}=\tilde{\Delta}_{0} \alpha\left(\tilde{P}_{\delta}\right)$ and $\tilde{\Delta}_{0} \alpha\left(\tilde{P}_{\sigma \cap \delta}\right)=\tilde{Q}_{\sigma^{\prime}} \tilde{Q}_{\delta^{\prime}}=$ $\tilde{Q}_{\sigma} \cap \delta^{\prime}$. This implies

$$
\hat{\alpha}(\hat{\sigma} \cap \hat{\delta})=\operatorname{supp}_{\mathscr{B}}\left(\alpha\left(\tilde{P}_{\sigma \cap \delta}\right)\right)=\hat{\sigma}^{\prime} \cap \hat{\delta}^{\prime}=\hat{\alpha}(\hat{\sigma}) \cap \alpha(\hat{\delta})
$$

and analogously $\hat{\alpha}(\hat{\sigma} \cup \hat{\delta})=\hat{\alpha}(\hat{\sigma}) \cup \hat{\alpha}(\hat{\delta}), \hat{\alpha}(\hat{\varnothing})=\hat{\varnothing}$ and $\hat{\alpha}(\hat{\mathbf{N}})=\hat{\mathbf{N}}$.

Isomorphism questions concerning the von Neumann algebras $D_{0} \mathscr{A}$ and $\Delta_{0} \mathscr{B}$ naturally arise. Clearly $D_{0} \mathscr{A}$ is isomorphic to $\Delta_{0} \mathscr{B}$ if and only 
if there exists a bijection $\tau_{0}: \mathbf{N} \rightarrow \mathbf{N}$ so that rank $P_{n}=\operatorname{rank} Q_{\tau(n)}$ for all $n$. In [5] J. Plastiras has shown that in fact $D_{0} \mathscr{A}+K(H)$ is isomorphic to $\Delta_{0} \mathscr{B}+K(H)$ if and only if there exists finite subsets $\mathbf{N}_{0}$ and $\mathbf{N}_{1}$ of $\mathbf{N}$ and a bijection $\tau_{0}: \mathbf{N} \backslash \mathbf{N}_{0} \rightarrow \mathbf{N} \backslash \mathbf{N}_{1}$ such that rank $\sum_{n \in \mathbf{N}_{0}} P_{n}=$ rank $\sum_{n \in \mathrm{N}_{1}} Q_{n}$ and, for all $n \in \mathbf{N} \backslash \mathbf{N}_{0}$, rank $P_{n}=Q_{\tau(n)}$. The results in this section give certain preliminary results concerning isomorphisms of $\tilde{D}_{0} \tilde{\mathscr{A}}$. A corollary of the proof of Lemma 2.3 yields such a result whenever $\left\{\text { rank } P_{n}\right\}_{n \in \mathbf{N}}$ is bounded. Whether this result is true in general appears to be an open question.

2.6. Proposition. Let $\left\{\text { rank } P_{n}\right\}_{n \in \mathbf{N}}$ be bounded. Then $\tilde{D}_{0} \tilde{\mathscr{A}}$ is isomorphic to $\tilde{\Delta}_{0} \tilde{\mathscr{B}}$ if and only if there exists finite subsets $\mathbf{N}_{0}$ and $\mathbf{N}_{1}$ of $\mathbf{N}$ and a bijection $\tau_{0}$ : $\mathbf{N} \backslash N_{0} \rightarrow N \backslash N_{1}$ such that for all $n \in \mathbf{N} \backslash \mathbf{N}_{1}$, rank $P_{n}$ $=\operatorname{rank} Q_{\tau(n)}$.

3. Restriction isomorphisms. Throughout this section $\omega, \gamma \in$ $N \backslash N_{0}$ will be fixed sets such that $\hat{\gamma}=\hat{\alpha}(\hat{\omega})$. The sequential representations

$$
\omega=\left\{a_{n}\right\}_{n \in \mathbf{N}}, \quad a_{n}<a_{n+1} ; \quad \gamma=\left\{\alpha_{n}\right\}_{n \in \mathbf{N}}, \quad \alpha_{n}<\alpha_{n+1},
$$

are unique. If we put

$$
P^{(\omega, n)}=P^{\left(a_{n}\right)}, \quad P_{1}^{\omega}=P_{\left[1, a_{1}\right]}, \quad P_{n+1}^{\omega}=P^{\left(a_{n+1}\right)}-P^{\left(a_{n}\right)}
$$

then the nest $\mathscr{N}_{A}^{\omega}=\left\{P^{(\omega, n)}\right\}_{n \in \mathrm{N}}$ determines the nest algebra $\mathscr{A}^{\omega}$. We shall define $D_{k}^{\omega}, \tilde{D}_{k}^{\omega}, \mathscr{A}_{0}^{\omega}, \tilde{\mathscr{A}}_{0}^{\omega}, \mathscr{A}_{(k)}^{\omega}, \tilde{\mathscr{A}}_{(k)}^{\omega}, k \geq 0$ as in $\S 1$. It is easy to see that $\tilde{\mathscr{A}}^{\omega}$ depends on $\hat{\omega}$ only and we have for $k \geq 0$

$$
\mathscr{A}^{\omega} \supset \mathscr{A}, \quad D_{k}^{\omega} \mathscr{A} \subset \mathscr{A}, \quad \mathscr{A}_{0}^{\omega} \subset \mathscr{A}_{0} \quad \text { and } \mathscr{A}_{(k)}^{\omega} \subset \mathscr{A}_{(k)} \text {. }
$$

For every $n \geq 1, \mathscr{A}_{(n)}^{\omega}$ is an ideal in $\mathscr{A}$ as well as in $\mathscr{A}^{\omega}$. Making analogous definitions for $\mathscr{B}$ and $\gamma$ we want to prove the relation $\alpha\left(\tilde{\mathscr{A}}_{(k)}^{\omega}\right)$ $=\tilde{\mathscr{B}}_{(k)}^{\gamma}, k \geq 0$. We shall use the notation

$$
\alpha^{\omega}: \tilde{\mathscr{A}} \rightarrow \tilde{\mathscr{B}}, \quad \text { where } \alpha^{\omega}(\tilde{A})=\alpha(\tilde{A}) \text { whenever } \tilde{A} \in \tilde{\mathscr{A}},
$$

to point out that we consider $\tilde{\mathscr{A}}$ and $\tilde{\mathscr{B}}$ as subalgebras in $\tilde{\mathscr{A}}^{\omega}$, respectively $\tilde{\mathscr{B}}^{\gamma}$ (which are related to the nests $\mathscr{N}_{\mathscr{\mathscr { A }}}^{\omega}$, respectively $\mathscr{N}_{\mathscr{\mathscr { B }}}^{\gamma}$ ). If $P=P^{2} \in \tilde{\mathscr{A}}$ then $D_{0}^{\omega} P$ is a projection in $\mathscr{A}\left(\subset \mathscr{A}^{\omega}\right)$ and we can define

$$
\operatorname{supp}_{\mathscr{A}^{\omega}}(P)=\left\{n \in N: P_{n}^{\omega} P P_{n}^{\omega} \neq 0\right\}
$$

and $\operatorname{supp}_{\mathscr{A}^{\omega}}(P)$ is determied by $\tilde{D}_{0}^{\omega} \tilde{P}$ modulo $\simeq$ (see Lemma 1.4). Thus if $\sigma \simeq \operatorname{supp}_{\mathscr{A}^{\omega}}(P)$ we shall put

$$
\operatorname{supp}_{\mathscr{A}^{\omega}}(\tilde{P})=\hat{\sigma} .
$$


Using Lemma 2.4 we can define

$$
\hat{\alpha}^{\omega}: \hat{\mathscr{N}} \rightarrow \hat{\mathscr{N}}
$$

by $\hat{\alpha}^{\omega}(\hat{\sigma})=\operatorname{supp}_{B^{\gamma}}(\alpha(\tilde{P}))$, if $\sigma \simeq \operatorname{supp}_{\mathscr{A}^{\omega}}(P)$ where $P=P^{2} \in \mathscr{A}$. It should be noted that $\hat{\alpha}^{\omega}$ depends on $\gamma$ and in fact different $\gamma$ give different values of $\hat{\alpha}^{\omega}$; however the argument in Lemma 4.10 shows that the resultant $\hat{\alpha}^{\omega}(\cdot)$ differ by a factor $\tau^{n_{0}}$ for some $n_{0}$.

For technical reasons we need to consider some compression algebras associated with $\mathscr{A}$ and subsets of $\mathbf{N}$. Let $P^{\prime}=P^{\prime 2} \in \mathscr{A}$. For simplicity assume that we have $\operatorname{supp}_{\mathscr{A}}\left(P^{\prime}\right)=\omega$. By Lemma 1.2 we know that $P^{\prime}$ is similar (in $\mathscr{A}$ ) to a self-adjoint projection $P^{\prime \prime} \in \mathscr{A}$. Let $\mathscr{A}_{p^{\prime}}, \mathscr{A}_{p^{\prime \prime}}$ be the compression algebras $P^{\prime} \mathscr{A} \mid P^{\prime} H$, resp. $P^{\prime \prime} \mathscr{A} \mid P^{\prime \prime} H$. If we put $P_{n}^{\prime \prime}=$ $P_{a_{n}} P^{\prime \prime} P_{a_{n}}, n \in N$, then $\mathscr{A}_{P^{\prime \prime}}$ appears to be the nest algebra determined by the nest $\left\{\sum_{k=1}^{n} P_{k}^{\prime \prime}\right\}_{n \in \mathbf{N}}$. This shows that $\mathscr{A}_{P^{\prime}}$ is a nest algebra. Choose $Q^{\prime} \in Q^{\prime 2} \in B$ such that $\tilde{Q}^{\prime}=\alpha\left(\tilde{P}^{\prime}\right)$ and $\operatorname{supp}_{B}\left(Q^{\prime}\right)=\gamma$. Now we can define the isomorphism

$$
\alpha_{P^{\prime}}: \tilde{\mathscr{A}}_{P^{\prime}} \rightarrow \tilde{\mathscr{B}}_{Q^{\prime}}
$$

as follows: Let $A^{\prime} \in \mathscr{A}_{P^{\prime}}$ and choose $B \in \alpha\left(\tilde{A}^{\prime} \tilde{P}^{\prime}\right)$ (where $A^{\prime} P^{\prime} \in \mathscr{A}$ ). Then putting $B^{\prime}=Q^{\prime} B \mid Q^{\prime} H$ we see that $\tilde{B}^{\prime}$ depends on $\tilde{A}^{\prime}$ only, thus $\alpha_{P^{\prime}}\left(\tilde{A}^{\prime}\right)=\tilde{B}^{\prime}$ is consistent and the properties of $\alpha_{P^{\prime}}$ can easily be checked. Since $\alpha_{P^{\prime}}$ is an isomorphism between essential nest algebras we can define $\hat{\alpha}_{P^{\prime}}$ as in $\S 2$.

$$
\begin{aligned}
& \text { 3.1. LEMmA. Let } P^{\prime}=P^{\prime 2} \in A, Q^{\prime} \in Q^{\prime 2} \text { be such that } \\
& \qquad \tilde{Q}^{\prime}=\alpha\left(\tilde{P}^{\prime}\right) \text { and } \operatorname{supp}_{\mathscr{A}}\left(P^{\prime}\right)=\operatorname{supp}_{\mathscr{B}}\left(Q^{\prime}\right)=N
\end{aligned}
$$

Then we have $\hat{\alpha}=\hat{\alpha}_{P^{\prime}}\left(\right.$ where $\left.\alpha_{P^{\prime}}: \tilde{\mathscr{A}}_{P^{\prime}} \rightarrow \tilde{\mathscr{B}}_{Q^{\prime}}\right)$.

Proof. It is plain that our statement is similarity stable thus we shall assume $P^{\prime}=P^{\prime *}, Q^{\prime}=Q^{\prime *}$. Let $\sigma \in \mathscr{N}$ and choose $Q=Q^{2} \in \mathscr{B}$ such that $\tilde{Q}=\alpha\left(\tilde{P}_{\sigma}^{\prime} \tilde{P}_{\sigma}\right), Q Q^{\prime}=Q^{\prime} Q=Q$. Making a new similarity we may suppose $Q=Q^{*}$ and $Q_{\delta}^{\prime}=Q \mid Q^{\prime} H$, where $\hat{\delta}=\hat{\alpha}_{P^{\prime}}(\hat{\sigma})$. But obviously $\hat{\sigma}$ is a minimal element in $\hat{\mathscr{N}}$, with the property $\alpha\left(\tilde{P}_{\sigma}\right) \tilde{Q}=\tilde{Q} \alpha\left(\tilde{P}_{\sigma}\right)=\tilde{Q}$ and this implies $\alpha(\hat{\sigma})=\hat{\alpha}_{p^{\prime}}(\hat{\sigma})$.

Recall that $\tau$ was defined as the set translation map on $\mathscr{N}$ with $\hat{\tau}$ the resultant map on $\hat{\mathscr{N}}$.

\subsection{THEOREM. $\hat{\alpha}$ commutes with $\hat{\tau}$.}

Proof. By Lemma 2.3 and Lemma 3.1 we may suppose rank $P_{n}=$ $\operatorname{rank} Q_{n}=1, n \in \mathbf{N}$. Let $\sigma \in \mathcal{N}$ and pick

$$
\sigma^{\prime} \in \hat{\tau}^{-1} \hat{\alpha}^{-1}(\hat{\alpha} \hat{\tau}(\hat{\sigma}) \backslash \hat{\tau} \hat{\alpha}(\hat{\sigma})) \quad \text { and } \quad \sigma^{\prime \prime} \in \hat{\alpha}^{-1} \hat{\tau}^{-1}(\hat{\tau} \hat{\alpha}(\hat{\sigma}) \backslash \hat{\alpha} \hat{\tau}(\hat{\sigma})) \text {. }
$$


Suppose $\hat{\sigma}^{\prime} \neq \hat{\varnothing}$. Since $\hat{\tau} \hat{\alpha}\left(\hat{\sigma}^{\prime}\right) \cap \hat{\alpha} \hat{\tau}\left(\hat{\sigma}^{\prime}\right)=\varnothing$, we can find $\delta \in \hat{\alpha} \hat{\tau}\left(\hat{\sigma}^{\prime}\right)$ and $\delta^{\prime} \in \hat{\alpha}\left(\hat{\sigma}^{\prime}\right)$ such that $\delta \cap \tau\left(\delta^{\prime}\right)=\varnothing$. Let $W \in \mathscr{A}_{(1)}$ be a partial isometry such that

$$
D_{1} W=W, \quad W^{*} W=P_{\tau\left(\sigma^{\prime}\right)} \quad \text { and } \quad W W^{*}=P_{\sigma^{\prime}} .
$$

By Theorem 2.2 we can find $U \in \mathscr{B}_{(1)}$ such that $\tilde{U}=\alpha(\tilde{W})$. Since we have

$$
\Delta_{0} U=0 \quad \text { and } \quad \tilde{U}=\alpha\left(\tilde{P}_{\sigma^{\prime}}\right) \tilde{U}=\tilde{U} \alpha\left(\tilde{P}_{\sigma^{\prime}}\right)
$$

we derive

$$
\tilde{\Delta}_{1} \tilde{U}=\left(\tilde{\Delta}_{0} \alpha\left(\tilde{P}_{\sigma^{\prime}}\right)\right)\left(\tilde{\Delta}_{1} \tilde{U}\right)=\left(\tilde{\Delta}_{1} \tilde{U}\right)\left(\tilde{\Delta}_{0} \alpha\left(\tilde{P}_{\sigma^{\prime}}\right)\right) .
$$

Because rank $Q_{n}=1$ for all $n \in \mathbf{N}$ we also have

$$
\tilde{\Delta}_{0} \alpha\left(\tilde{P}_{\sigma^{\prime}}\right)=\tilde{Q}_{\delta^{\prime}}, \quad \tilde{\Delta}_{0} \alpha\left(\tilde{P}_{\tau\left(\sigma^{\prime}\right)}\right)=\tilde{Q}_{\delta}, \quad \mathscr{B}_{(2)}=\left(\mathscr{B}_{(1)}\right)^{2}
$$

and in particular

$$
\tilde{\Delta}_{1} \tilde{U}=\tilde{Q}_{\delta^{\prime}}\left(\tilde{\Delta}_{1} \tilde{U}\right)=\left(\tilde{\Delta}_{1} \tilde{U}\right) \tilde{Q}_{\delta}
$$

Now using the obvious relation $Q_{\delta^{\prime}}\left(\Delta_{1} U\right)=\left(\Delta_{1} U\right) Q_{\tau\left(\delta^{\prime}\right)}$ we infer

$$
\tilde{\Delta}_{1} \tilde{U}=\tilde{Q}_{\delta^{\prime}}\left(\tilde{\Delta}_{1} \tilde{U}\right) \tilde{Q}_{\delta}=\left(\tilde{\Delta}_{1} \tilde{U}\right) \tilde{Q}_{\tau\left(\delta^{\prime}\right)} \tilde{Q}_{\delta}=0
$$

or equivalently $\tilde{U} \in \tilde{B}_{(2)}$. Let $U_{1}, U_{2} \in \mathscr{B}_{(1)}$ be such that $\tilde{U}=\tilde{U}_{1} \tilde{U}_{2}$. Since by Theorem 2.2 we have

$$
\tilde{W}=\alpha^{-1}\left(\tilde{U}_{1}\right) \alpha^{-1}\left(U_{2}\right) \in\left(\tilde{\mathscr{A}}_{(1)}\right)^{2}=\mathscr{A}_{(2)},
$$

and this is a contradiction, we deduce $\hat{\sigma}^{\prime}=\hat{\varnothing}$ and analogously $\hat{\sigma}^{\prime \prime}=\hat{\varnothing}$. It follows that $\hat{\alpha} \hat{\tau}=\hat{\tau} \hat{\alpha}$.

3.3. Lemma. Let $\tilde{A}=\tilde{A}^{2} \in \tilde{\mathscr{A}}$. Then $\tilde{A} \in \tilde{\mathscr{A}}_{(0)}^{\omega}$ if and only if $\tilde{D}_{0} \tilde{A} \in \tilde{\mathscr{A}}_{0}^{\omega}$. Moreover if $\tilde{A} \in \tilde{\mathscr{A}}_{(0)}^{\omega}$ then $\tilde{D}_{0}^{\omega} \tilde{A}=\tilde{D}_{0} \tilde{A}$.

Proof. Suppose $\tilde{A} \in \tilde{\mathscr{A}}_{(0)}^{\omega}$. Using Lemma 1.3 we can find $P=P^{2} \in$ $\mathscr{A}_{(0)}^{\omega}$ such that $\tilde{P}=\tilde{A}$ and in particular $D_{0}^{\omega} P=P_{\sigma}^{\omega}$ for some $\sigma \in \mathscr{N}$. Since obviously $P_{n}^{\omega} \in A_{0}, n \in \mathrm{N}$ and $D_{0} D_{0}^{\omega} P=D_{0}^{\omega} D_{0} P=D_{0} P$, we derive

$$
D_{0} P=D_{0} D_{0}^{\omega} P=D_{0} P_{\sigma}^{\omega}=P_{\sigma}^{\omega} \in A_{0}^{\omega} \text {. }
$$

If $\tilde{D}_{0} \tilde{A} \in \tilde{\mathscr{A}}_{0}^{\omega}$, applying again Lemma 1.3 we can find $P=P^{2} \in \mathscr{A}_{(0)}$ such that $\tilde{P}=\tilde{A}$ and $D_{0} P=P_{\sigma}^{\omega}$ for some $\sigma \in \mathcal{N}$. Since $P_{n}^{\omega}-P_{n}^{\omega} P P_{n}^{\omega}$, $n \in \mathbf{N}$ is a projection and $D_{0}\left(P_{n}^{\omega}-P_{n}^{\omega} P P_{n}^{\omega}\right)=0, n \in \sigma$, we easily derive $P_{n}^{\omega}=P_{n}^{\omega} P P_{n}^{\omega}, n \in \sigma$. If $n \in \mathbf{N} \backslash \sigma$, then $P_{n}^{\omega} P P_{n}^{\omega}$ is a projection such that 
$D_{0}\left(P_{n}^{\omega} P P_{n}^{\omega}\right)=0$, consequently $P_{n}^{\omega} P P_{n}^{\omega}=0$. Thus it follows:

$$
D_{0}^{\omega} P=\sum_{n \in \mathbf{N}} P_{n}^{\omega} P P_{n}^{\omega}=D_{0} P \in \mathscr{A}_{0}^{\omega},
$$

or equivalently $P \in \mathscr{A}_{(0)}^{\omega}$.

3.4. Lemma. Let $\tilde{A}=\tilde{A}^{2} \in \tilde{\mathscr{A}}_{(0)}, P=P^{2} \in \tilde{A}$ and let $\sigma \in \operatorname{supp}_{\mathscr{A}}(\tilde{P})$ be of the form $\sigma=\bigcup_{n \in \mathrm{N}}\left[b_{n}, c_{n}\right], b_{n} \leq c_{n}<b_{n+1}$. Let us put $\sigma_{l}=\left\{b_{n}\right\}_{n \in \mathrm{N}}$, $\sigma_{r}=\left\{c_{n}\right\}_{n \in \mathbf{N}}$. Then we have $\tilde{A} \in \tilde{\mathscr{A}}_{(0)}^{\omega}$ if and only if $\hat{\tau}^{-1}\left(\hat{\sigma}_{l}\right) \cup \hat{\sigma}_{r} \subset \hat{\omega}$.

Proof. Since the part "only if" is obvious assume $\hat{\tau}^{-1}\left(\hat{\sigma}_{l}\right) \cup \hat{\sigma}_{r} \subset \hat{\omega}$. After deleting a finite set we can reduce to the case $\sigma \subset \tau(\mathbf{N}), \tau^{*}\left(\sigma_{l}\right) \cup \sigma_{r}$ $\subset \omega$. But this obviously implies $P_{\sigma} \in \mathscr{A}_{0}^{\omega}$ and because $\tilde{D}_{0} \tilde{P}=\tilde{P}_{\sigma}$, applying Lemma 3.3 we infer $\tilde{A} \in \tilde{\mathscr{A}}_{(0)}^{\omega}$.

\subsection{Lemma. Let $\tilde{A} \in \tilde{\mathscr{A}}$. Then $\tilde{A} \in \tilde{\mathscr{A}}_{(0)}^{\omega}$ iff $\tilde{D}_{0} \tilde{A}=\tilde{D}_{0}^{\omega} \tilde{A} \in \tilde{\mathscr{A}}_{0}^{\omega}$.}

Proof. If $\tilde{A} \in \tilde{\mathscr{A}}_{(0)}^{\omega}$ we may suppose that there exists $A \in \tilde{\mathscr{A}}$ of the form $A=A_{0}+A_{1}$, where $A_{0} \in \mathscr{A}_{0}^{\omega}, A_{1} \in \mathscr{A}_{(1)}^{\omega}$. Because $\mathscr{A}_{0}^{\omega} \subset \mathscr{A}_{0}$ and $A_{(1)}^{\omega} \subset \mathscr{A}_{(1)}$ we deduce

$$
D_{0}^{\omega} A=A_{0}=D_{0} A_{0}=D_{0} A \in \mathscr{A}_{0}^{\omega} .
$$

The part "if" is trivial.

\subsection{Lemma. For every $\tilde{A}=\tilde{A}^{2} \in \mathscr{A}_{(0)}^{\omega}$ we have $\alpha(\tilde{A}) \in \tilde{\mathscr{B}}_{(0)}^{\gamma}$.}

Proof. Pick $\delta \in \operatorname{supp}_{\mathscr{B}}(\alpha(\tilde{A}))$ and define $\delta_{l}, \delta_{r}$ as in Lemma 3.6. Since obviously $\hat{\tau}^{-1}\left(\hat{\delta}_{l}\right) \cap \hat{\delta}=\hat{\varnothing}$, using Theorem 3.2 we deduce $\hat{\tau}^{-1} \hat{\alpha}^{-1}\left(\hat{\delta}_{l}\right) \cap \hat{\alpha}^{-1}(\hat{\delta})=\hat{\varnothing}$. Let $\sigma \in \hat{\alpha}^{-1}(\hat{\delta})$ and $\sigma^{\prime} \in \hat{\alpha}^{-1}\left(\hat{\delta}_{l}\right)$. Since $\sigma \in$ $\operatorname{supp}_{\mathscr{A}}(\tilde{A}), \tilde{A} \in \tilde{\mathscr{A}}_{(0)}^{\omega}$ and $\hat{\boldsymbol{\sigma}}^{\prime} \subset \hat{\boldsymbol{\sigma}}, \hat{\tau}^{-1}\left(\hat{\sigma}^{\prime}\right) \cap \hat{\boldsymbol{\sigma}}=\hat{\varnothing}$ we easily derive $\hat{\boldsymbol{\sigma}}^{\prime} \subset$ $\hat{\sigma}_{l}, \hat{\tau}^{-1}\left(\hat{\sigma}^{\prime}\right) \subset \hat{\omega}$. It follows $\hat{\tau}^{-1}\left(\hat{\delta}_{l}\right) \subset \hat{\gamma}$ and analogously $\hat{\delta}_{r} \subset \hat{\gamma}$, thus by Lemma 3.4, $\alpha(\tilde{A}) \in \tilde{\mathscr{B}}_{(0)}^{\gamma}$.

\subsection{THEOREM. $\alpha\left(\tilde{\mathscr{A}}_{(0)}^{\omega}\right)=\tilde{\mathscr{B}}_{(0)}^{\gamma}$.}

Proof. Let $A \in \mathscr{A}_{(0)}^{\omega}, B \in \mathscr{B}_{(0)}$ be such that $\tilde{B}=\alpha(\tilde{A})$. Suppose $\tilde{B} \notin \tilde{\mathscr{B}}_{(0)}^{\gamma}$. Then we can choose $\lambda_{n} \in \sigma\left(Q_{n}^{\gamma} B \mid Q_{n}^{\gamma} H\right)$ with

$$
\lim _{n \rightarrow \infty}\left\|Q_{n}^{\gamma}\left(B-\lambda_{n}\right) Q_{n}^{\gamma}\right\|>0 \text {. }
$$

Applying Lemma 1.5 we can find $\delta \in \mathscr{N} \backslash \mathscr{N}_{0}$ such that

$$
\begin{aligned}
& \lim _{n \in \delta} \lambda_{n}=\lambda, \quad \tilde{Q}_{\delta}^{\gamma} \tilde{B} \tilde{Q}_{\delta}^{\gamma}=\tilde{Q}_{\delta}^{\gamma}\left(\tilde{\Delta}_{0}^{\gamma} \tilde{B}\right) \tilde{Q}_{\delta}^{\gamma}, \\
& \left\|Q_{n}^{\gamma}(B-\lambda) Q_{n}^{\gamma}\right\| \geq a>0, \quad n \in \delta .
\end{aligned}
$$


Since by Lemma 3.6 we have $\alpha^{-1}\left(\tilde{Q}_{\delta}^{\gamma}\right) \tilde{A} \alpha^{-1}\left(\tilde{Q}_{\delta}^{\gamma}\right) \in \mathscr{A}_{(0)}^{\omega}$ we can use again Lemma 1.5 to produce $\sigma \in \mathscr{N} \backslash \mathscr{N}_{0}$ such that

$$
\tilde{P}_{\sigma}^{\omega}\left(\tilde{D}_{0}^{\omega} \alpha^{-1}\left(\tilde{Q}_{\delta}^{\gamma}\right)\right)=\tilde{P}_{\sigma}^{\omega} \quad \text { and } \quad \tilde{P}_{\sigma}^{\omega} \tilde{A} \tilde{P}_{\sigma}^{\omega}=\mu \tilde{P}_{\sigma}^{\omega} .
$$

Now applying Lemma 3.6 we can find $\delta^{\prime} \in \mathscr{N} \backslash \mathscr{N}_{0}$ and $\delta^{\prime} \subset \delta$ such that

$$
\tilde{\Delta}_{0}^{\gamma} \alpha\left(\tilde{P}_{\sigma}^{\omega}\right)=\tilde{Q}_{\delta^{\prime}}^{\gamma} \quad \text { and } \quad \tilde{Q}_{\delta^{\prime}}^{\gamma}\left(\tilde{\Delta}_{0}^{\gamma} \tilde{B}\right) \tilde{Q}_{\delta^{\prime}}^{\gamma}=\mu \tilde{Q}_{\delta^{\prime}}^{\gamma}
$$

But because $\lambda_{n} \in \sigma\left(Q_{n}^{\gamma} B \mid Q_{n}^{\gamma} H\right)$ we deduce that

$$
\lim _{n \in \delta^{\prime}} \lambda_{n}=\lambda=\mu \quad \text { and }\left\|Q_{n}^{\gamma}(B-\mu) Q_{n}^{\gamma}\right\| \geq \frac{a}{2}
$$

for some $n \geq n_{0}, n \in \delta^{\prime}$

which yields a contradiction. It therefore follows that $\alpha\left(\tilde{\mathscr{A}}_{(0)}^{\omega}\right) \subset \tilde{\mathscr{B}}_{(0)}^{\gamma}$ and analogously $\alpha^{-1}\left(\tilde{\mathscr{B}}_{(0)}^{\gamma}\right) \subset \tilde{\mathscr{A}}_{(0)}^{\omega}$.

3.8. CoROllaRy. The compression $\tilde{\Delta}_{0}^{\gamma} \alpha^{\omega}: \tilde{\mathscr{A}}_{0}^{\omega} \rightarrow \tilde{\mathscr{B}}_{0}^{\gamma}$ is an isomorphism with

$$
\begin{gathered}
\left(\tilde{\Delta}_{0}^{\gamma} \alpha^{\omega} \mid \tilde{\mathscr{A}}_{0}^{\omega}\right)=\left(\tilde{\Delta}_{0}^{\omega} \alpha \mid \mathscr{A}_{0}^{\omega}\right) \\
\left(\tilde{\Delta}_{0}^{\gamma} \alpha^{\omega} \mid \tilde{\mathscr{A}}_{0}^{\omega}\right)^{-1}=\left(\tilde{D}_{0}^{\omega} \alpha^{-1} \mid \tilde{\mathscr{B}}_{0}^{\gamma}\right) \quad \text { and } \quad \alpha\left(\tilde{\mathscr{A}}_{(1)}^{\omega}\right)=\tilde{\mathscr{B}}_{(1)}^{\gamma} .
\end{gathered}
$$

Proof. We apply Lemma 2.1 and Theorem 3.7.

3.9. LEMMA. Let $P^{\prime}=P^{\prime 2} \in \mathscr{A}, Q^{\prime}=Q^{\prime 2} \in \mathscr{B}$ be such that $\tilde{Q}^{\prime}=$ $\alpha\left(\tilde{P}^{\prime}\right), \operatorname{supp}_{\mathscr{A}}\left(P^{\prime}\right)=\left\{a_{n}^{\prime}\right\}_{n \in \mathbf{N}}, \operatorname{supp}_{\mathscr{B}}\left(Q^{\prime}\right)=\left\{\alpha_{n}^{\prime}\right\}_{n \in \mathbf{N}}$ where $a_{n-1}<a_{n}^{\prime}$ $\leq a_{n}$ and $\alpha_{n-1}<\alpha_{n}^{\prime} \leq \alpha_{n}, n \in \mathbf{N}$. Then we have $\hat{\alpha}^{\omega}=\hat{\alpha}_{P^{\prime}}$ (where $\alpha_{P^{\prime}}$ : $\left.\tilde{\mathscr{A}}_{P^{\prime}} \rightarrow \tilde{\mathscr{B}}_{Q^{\prime}}\right)$.

Proof. Let $\sigma \in \mathcal{N}$. By Theorem 3.7 we know that we have $\tilde{\Delta}_{0}^{\gamma} \alpha^{\omega}\left(\tilde{P}_{\sigma}^{\omega}\right)$ $=\tilde{Q}_{\delta}^{\gamma}$ where $\delta \in \operatorname{supp}_{\mathscr{B}^{\gamma}}\left(\alpha\left(\tilde{P}_{\sigma}^{\omega}\right)\right)$. Let $Q=Q^{2} \in \mathscr{B}$ be such that $Q Q^{\prime}=$ $Q^{\prime} Q=Q$ and $\tilde{Q}=\alpha\left(P_{\sigma}^{\prime} \tilde{P}_{\sigma}^{\omega}\right)$. Since $\hat{\sigma}$ is minimal in $\hat{\mathscr{N}}$, with the property $\alpha\left(\tilde{P}_{\sigma}^{\omega}\right) \tilde{Q}=\tilde{Q} \alpha\left(P_{\sigma}^{\omega}\right)=\tilde{Q}$ we derive as in the proof of Lemma 3.1, that we have $\hat{\alpha}_{p^{\prime}}(\hat{\boldsymbol{\sigma}})=\hat{\delta}=\hat{\alpha}^{\omega}(\hat{\boldsymbol{\sigma}})$.

3.10. CoRollary. $\hat{\alpha}^{\omega}$ is a lattice isomorphism and $\hat{\alpha}^{\omega} \hat{\tau}=\hat{\tau} \hat{\alpha}^{\omega}$.

Proof. Apply Theorem 3.2 and Lemma 3.9.

3.11. Lemma. For every $\sigma \in \mathscr{N} \backslash \mathscr{N}_{0}, \delta \in \hat{\alpha}^{\omega}(\sigma)$ put $\omega_{\sigma}=\left\{a_{n}\right\}_{n \in \sigma}$, $\gamma_{\delta}=\left\{\alpha_{n}\right\}_{n \in \delta}$. Then we have

$$
\hat{\alpha}\left(\hat{\omega}_{\sigma}\right)=\hat{\gamma}_{\delta} .
$$


Proof. Making a similarity we may suppose that we have $\tilde{Q}_{\gamma}=\alpha\left(\tilde{P}_{\omega}\right)$ and $\tilde{Q}^{\prime}=\alpha\left(\tilde{P}_{\omega_{\sigma}}\right)$ where $Q^{\prime} Q_{\gamma}=Q_{\gamma} Q^{\prime}=Q^{\prime}$. Let $\mathscr{A}^{\prime}, \mathscr{B}^{\prime}$ denote the compression algebras $\mathscr{A}_{P_{\omega}}$, resp. $\mathscr{B}_{Q_{\gamma}}$ and let

$$
\alpha^{\prime}: \tilde{\mathscr{A}}^{\prime} \rightarrow \tilde{\mathscr{B}}^{\prime}
$$

denote the isomorphism induced by $\alpha$. Since the block-diagonal projections of $\mathscr{B}^{\prime}$ are $\left\{Q_{\alpha_{n}}\right\}_{n \in \mathrm{N}}$ and by Lemma 3.9 we have $\operatorname{supp}_{\mathscr{B}^{\prime}} Q^{\prime} \simeq \delta$ we deduce $\operatorname{supp}_{B} Q^{\prime} \simeq \gamma_{\delta}$ or equivalently $\hat{\alpha}\left(\hat{\omega}_{\sigma}\right)=\hat{\gamma}_{\delta}$.

3.12. Proposition. For every $k \geq 0$ we have $\alpha\left(\tilde{\mathscr{A}}_{(k)}^{\omega}\right)=\tilde{\mathscr{B}}_{(k)}^{\gamma}$.

Proof. For $k=0$ we apply Theorem 3.7. If $k>0$ put

$$
\sigma_{m}=\{k n+m: n \in \mathbf{N}\}, \quad 0 \leq m<k
$$

and pick $\delta_{m} \in \hat{\alpha}^{\omega}\left(\hat{\sigma}_{m}\right)$. Since $\sigma_{m}=\tau^{m}\left(\sigma_{0}\right)$, Corollary 3.10 implies $\hat{\delta}_{m}=$ $\hat{\tau}\left(\hat{\delta}_{0}^{m}\right)$ and by Lemma 3.11 we derive $\hat{\gamma}_{\delta_{m}}=\hat{\alpha}\left(\hat{\omega}_{\sigma_{m}}\right)$. On the other hand because $\sigma_{m} \cap \sigma_{i}=\varnothing, m \neq i$ and $\bigcup_{m=0}^{k-1} \sigma_{m}=\mathbf{N}$ we infer that $\delta_{m}$ can be chosen of the form

$$
\delta_{m}=\left\{k\left(n+n_{0}\right)+m: n \in \mathbf{N}\right\} \quad \text { for some } n_{0} \in \mathbf{N} .
$$

But using the relations

$$
\tilde{A}_{(k)}^{\omega}=\bigcap_{m=0}^{k-1} \tilde{A}_{(1)}^{\omega_{m}}, \quad \tilde{B}_{(k)}^{\gamma_{m}}=\bigcap_{m=0}^{k-1} \tilde{B}_{(1)}^{\gamma_{m}}, \quad \text { where } \omega_{m}=\omega_{\sigma_{m}}, \gamma_{m}=\gamma_{\delta_{m}}
$$

and because by Corollary 3.8 we have $\alpha\left(\tilde{\mathscr{A}}_{(1)}^{\omega_{m}}\right)=\tilde{\mathscr{B}}_{(1)}^{\gamma_{m}}$, the proof is concluded.

3.13. LeMMA. Let $\sigma \in \mathcal{N}, \delta \in \hat{\alpha}^{\omega}(\hat{\sigma})$ and let $\sigma_{l}, \delta_{l}, \sigma_{r}, \delta_{r}$ be defined as in Lemma 3.4. Then we have

$$
\hat{\delta}_{l}=\hat{\alpha}^{\omega}\left(\hat{\sigma}_{l}\right) \quad \text { and } \quad \hat{\delta}_{r}=\hat{\alpha}^{\omega}\left(\hat{\sigma}_{r}\right) .
$$

Proof. We have $\hat{\alpha}^{\omega}\left(\hat{\sigma}_{l}\right) \subset \hat{\delta}$ and by Corollary 3.10, we deduce

$$
\hat{\boldsymbol{\tau}}^{-1} \hat{\boldsymbol{\alpha}}^{\omega} \hat{\tau}^{-1}\left(\hat{\boldsymbol{\sigma}}_{l}\right) \subset \hat{\mathbf{N}} \backslash \hat{\boldsymbol{\delta}}
$$

which implies $\hat{\alpha}^{\omega}\left(\hat{\sigma}_{l}\right) \subset \hat{\delta}_{l}$. Analogously we can prove that we have $\hat{\alpha}^{\omega}\left(\hat{\sigma}_{l}\right)$ $\supset \hat{\delta}_{l}$ and $\hat{\alpha}^{\omega}\left(\hat{\sigma}_{r}\right)=\hat{\delta}_{r}$.

4. The Essential Equivalence of $\mathscr{N}_{A}$ and $\mathscr{N}_{B}$. The aim of this section is to prove the main result of the paper, namely:

4.1. THEOREM. There exist $n_{0} \in \mathbf{N}$ and an integer $m_{0}$ such that $\hat{\alpha}=\hat{\tau}^{m_{0}} \quad$ and $\quad \operatorname{rank} P_{n}=\operatorname{rank} Q_{n+m_{0}} \quad$ whenever $n \geq n_{0}$. 
The second relation in the above statements describes what "essential equivalence of $\mathscr{N}_{\mathscr{A}}$ and $\mathscr{N}_{\mathscr{B}}$ " means.

In he sequel we need some new notation. Thus we consider the ideals (in $\mathscr{A}) I_{\mathscr{A}}^{\omega}, F_{\mathscr{A}}^{\omega}$ defined as follows.

$A \in I_{\mathscr{A}}^{\omega}$ if $A \in \mathscr{A}_{(1)}^{\omega}$ and for each $n \in \mathrm{N}$ all entries of $A$ along the $n$th diagonal in $\mathscr{A}^{\omega}$, except the first $n(n+1) / 2$, are 0 .

$A \in F_{\mathscr{A}}^{\omega}$ if $A \in \mathscr{A}_{(2)}^{\omega}$ and for each $n \in N, n \geq 2$ all entries of $A$ along the $n$th diagonal in $\mathscr{A}^{\omega}$, except the first $(n-1) n / 2$, are 0 .

To be more rigorous, let $[a]$ denote the integer part of $a \in \mathbf{R}$ and define the function $v: \mathbf{N} \rightarrow \mathbf{N}$ by

$$
v(n)=\left[\frac{1+\sqrt{8 n-7}}{2}\right]+n, \quad n \in \mathbf{N} .
$$

It is easy to see that we have

$$
v(n)=k+n \quad \text { whenever } \frac{(k-1) k}{2}<n \leq \frac{k(k+1)}{2} \quad \text { for } k \in \mathbf{N} .
$$

We define the ideals $I_{\mathscr{A}}^{\omega}, F_{\mathscr{A}}^{\omega}$ by

$$
\begin{aligned}
& I_{\mathscr{A}}^{\omega}=\left\{A \in L(H):\left(I-P^{(\omega, n-1)}\right) A P^{(\omega, v(n)-1)}=0, n \in N\right\}, \\
& F_{\mathscr{A}}^{\omega}=\left\{A \in L(H):\left(I-P^{(\omega, n-1)}\right) A P^{(\omega, v(n))} 0, n \in N\right\},
\end{aligned}
$$

where $P^{(\omega, 0)}=0$.

Applying Lemma 1.1 we note that $\tilde{I}_{\mathscr{A}}^{\omega}$ and $\tilde{F}_{\mathscr{A}}^{\omega}$ are closed ideals in $\tilde{\mathscr{A}}$.

4.2. LemMA. Let $P, P^{\prime} \in \mathscr{A}$ be selfadjoint projections and let $W \in D_{k}^{\omega} \mathscr{A}$ $(k \geq 1)$ be such that

$$
P^{\prime} W: P H \rightarrow P^{\prime} H
$$

is a Fredholm operator. Let $Q=Q^{2} \in \mathscr{B}, Q^{\prime}=Q^{\prime 2} \in \mathscr{B}, \tilde{Q}=\alpha(\tilde{P})$, $\tilde{Q}^{\prime}=\alpha\left(\tilde{P}^{\prime}\right), U \in \alpha(\tilde{W})$ and put

$$
U_{k}=\left(\Delta_{0}^{\gamma} Q^{\prime}\right)\left(\Delta_{k}^{\gamma} U\right):\left(\Delta_{0}^{\gamma} Q\right) H \rightarrow\left(\Delta_{0}^{\gamma} Q^{\prime}\right) H .
$$

Then $U_{k}$ is a Fredholm operator.

Proof. Suppose that $U_{k}$ is not left essentially invertible. Since by Proposition 3.12 we have $\tilde{U} \in \tilde{\mathscr{B}}_{(k)}^{\gamma}$, it is easy to find $B \in \Delta_{0}^{\gamma} \mathscr{B}$, such that

$$
\left(\Delta_{0}^{\gamma} Q\right) B=B, \quad \tilde{B} \neq 0, \quad \tilde{Q}^{\prime} \tilde{U} \tilde{B} \in \tilde{\mathscr{B}}_{(k+1)}^{\gamma} .
$$


Because $Q B-B \in \mathscr{B}_{(1)}^{\gamma}$, using Corollary 3.8 and Proposition 3.12 we deduce

$$
\tilde{P}\left(\tilde{D}_{0}^{\omega} \alpha^{-1}(\tilde{B})\right)=\tilde{D}_{0}^{\omega} \alpha^{-1}(\tilde{B}) \neq 0 \quad \text { and } \quad \tilde{P}^{\prime} \tilde{W} \alpha^{-1}(\tilde{B}) \in \tilde{\mathscr{A}}_{(k+1)}^{\omega} .
$$

But this implies $\tilde{P}^{\prime} \tilde{W} \tilde{P}\left(\tilde{D}_{0}^{\omega} \alpha^{-1}(\tilde{B})\right)=0$, contradicting our hypothesis. Analogously we prove that $U_{k}$ is right essentially invertible.

4.3. LemMA. Let us put $\omega_{0}=\{n(n+1) / 2\}_{n \in \mathrm{N}}$. Then there exists an integer $m_{0}$ such that

$$
\hat{\alpha}^{\omega}\left(\hat{\omega}_{0}\right)=\hat{\tau}^{m_{0}}\left(\hat{\omega}_{0}\right)
$$

Proof. By Lemma 3.9 we may and we shall suppose $\omega=\mathbf{N}$, thus $\alpha^{\omega}=\alpha$, and $\operatorname{rank} P_{n}=\operatorname{rank} Q_{n}=1, n \in \mathbf{N}$. Suppose that $\gamma_{0}=\alpha\left(\omega_{0}\right)$ is of the form $\gamma_{0}=\left\{\beta_{n}\right\}_{n \in \mathbf{N}}, \beta_{n}<\beta_{n+1}$. Since rank $P_{n}^{\omega_{0}}=n$ we can find $W \in D_{1}^{\omega_{0}} \mathscr{A}$ such that $W W^{*}=I, W^{*} W=I-P_{\omega_{0}}$. Applying Lemma 1.2 and Proposition 3.12 we reduce to the case

$$
\tilde{U}=\alpha(\tilde{W}), \quad U \in B_{(1)}^{\gamma_{0}}, \quad \tilde{Q}_{\gamma_{0}}=\alpha\left(\tilde{P}_{d \omega_{0}}\right), \quad U\left(I-Q_{\gamma_{0}}\right)=U .
$$

Since in particular $\left(\Delta_{1}^{\gamma_{0}} U\right)\left(I-Q_{\gamma_{0}}\right)=\Delta_{1}^{\gamma_{0}} U$ and by Lemma 4.2.

$$
\Delta_{1}^{\gamma_{0}} U:\left(I-Q_{\gamma_{0}}\right) H \rightarrow H
$$

is a Fredholm operator, we can find $n_{0} \in \mathbf{N}$, such that

$$
\operatorname{rank}\left(Q_{n+1}^{\gamma_{0}}-Q_{\beta_{n+1}}\right)=\operatorname{rank} Q_{n}^{\gamma_{0}}, \quad n \geq n_{0} .
$$

Now using the fact that rank $Q_{n}^{\gamma_{0}}=\beta_{n}-\beta_{n-1}$, we deduce

$$
\begin{aligned}
\beta_{n_{0}+n}-\beta_{n_{0}} & =\sum_{k=1}^{n}\left(k+r_{0}\right) \\
& =-\frac{r_{0}\left(r_{0}+1\right)}{2}+\frac{\left(r_{0}+n\right)\left(r_{0}+n+1\right)}{2}, \quad n \in N,
\end{aligned}
$$

where $r_{0}=\operatorname{rank} Q_{n_{0}}^{\gamma_{0}}$. Thus putting $m_{0}=\beta_{n_{0}}-r_{0}\left(r_{0}+1\right) / 2$ we have

$$
\beta_{n_{0}+n}=m_{0}+\frac{\left(r_{0}+n\right)\left(r_{0}+n+1\right)}{2}, \quad n \in \mathbf{N}
$$

or equivalently $\hat{\gamma}_{0}=\hat{\alpha}\left(\omega_{0}\right)=\hat{\tau}^{m_{0}}\left(\hat{\omega}_{0}\right)$.

Let $v$ be the function introduced in the beginning of this section and define $\hat{v}: \hat{\mathscr{N}} \rightarrow \hat{\mathscr{N}}$ by

$$
\hat{v}(\hat{\sigma})=\widehat{v(\sigma)} \quad \text { whenever } \sigma \in \mathscr{N} .
$$


4.4. LeMMA. Let $\omega_{0}$ be as in Lemma 4.3 and assume $\hat{\omega}_{0}=\hat{\alpha}^{\omega}\left(\hat{\omega}_{0}\right)$. Then $\hat{\alpha}^{\omega}$ commutes with $\hat{v}$.

Proof. as in Lemma 4.3 we shall replace $\alpha^{\omega}$ by $\alpha$, we shall assume rank $P_{n}=\operatorname{rank} Q_{n}=1, n \in \mathbf{N}$, and then to simplify the notation we shall also assume $\omega=\omega_{0}$. This will allow us to take $\hat{\gamma}=\hat{\omega}$. Now suppose that our Lemma is false. Then proceeding as in the proof of Theorem 3.2 we can find $\sigma \in \mathscr{N}$ of the form $\sigma=\left\{r_{n}\right\}_{n \in \mathbf{N}}$ and $s_{n} \in \mathbf{N}$ such that

$$
\frac{\left(s_{n}-1\right) s_{n}}{2}<r_{n} \leq \frac{s_{n}\left(s S_{n}+1\right)}{2}, \quad s_{n}+1 \leq s_{n+1}, \quad n \in \mathbf{N}
$$

and $\hat{\alpha} \hat{v}(\hat{\sigma}) \cap \hat{v} \hat{\alpha}(\hat{\sigma})=\hat{\varnothing}$. By the definition of $v$ we easily derive

$$
v\left(\frac{\left(s_{n}-1\right) s_{n}}{2}\right)<v\left(r_{n}\right) \leq v\left(\frac{s_{n}\left(s_{n}+1\right)}{2}\right), \quad v\left(r_{n}\right)=s_{n}+r_{n}
$$

and

$$
v\left(\frac{s_{n}\left(s_{n}+1\right)}{2}\right)=\frac{\left(s_{n}+1\right)\left(s_{n}+2\right)}{2}-1 .
$$

Consequently

$$
\frac{s_{n}\left(s_{n}+1\right)}{2}-r_{n}=v\left(\frac{s_{n}\left(s_{n}+1\right)}{2}\right)-v\left(r_{n}\right) .
$$

Let $\sigma^{\prime} \in \mathscr{N}$ be such that $\omega_{\sigma^{\prime}}=\left\{s_{n}\left(s_{n}+1\right) / 2\right\}_{n \in \mathbf{N}}$ and pick $\delta^{\prime} \in \hat{\alpha}^{\omega}\left(\sigma^{\prime}\right)$. Applying Corollary 3.10 and Lemma 3.11 we infer

$$
\hat{\omega}_{\delta^{\prime}}=\hat{\alpha}\left(\hat{\omega}_{\sigma^{\prime}}\right) \text { and } \quad \hat{\omega}_{\tau\left(\delta^{\prime}\right)}=\hat{\alpha}\left(\hat{\omega}_{\tau\left(\sigma^{\prime}\right)}\right) .
$$

Let us put

$$
\begin{gathered}
\sigma_{n}^{(1)}=\left[r_{n}, \frac{s_{n}\left(s_{n}+1\right)}{2}\right], \quad \sigma_{n}^{(2)}=\left[v\left(r_{n}\right), \frac{\left(s_{n}+1\right)\left(s_{n}+2\right)}{2}-1\right], \\
\sigma^{(1)}=\bigcup_{n \in \mathbf{N}} \sigma_{n}^{(1)}, \quad \sigma^{(2)}=\bigcup_{n \in \mathbf{N}} \sigma_{n}^{(2)}
\end{gathered}
$$

and pick $\delta^{(1)} \in \hat{\alpha}\left(\hat{\sigma}^{(1)}\right), \delta^{(2)} \in \hat{\alpha}\left(\hat{\sigma}^{(2)}\right)$. If $\omega_{\delta^{\prime}}=\left\{s_{n}^{\prime}\left(s_{n}^{\prime}-1\right) / 2\right\}_{n \in \mathbf{N}}$ then applying Lemma 3.13 we may suppose that we have $\delta^{(1)}=\bigcup_{n \in \mathrm{N}} \delta_{n}^{(1)}$, $\delta^{(2)}=\bigcup_{n \in \mathrm{N}} \delta_{n}^{(2)}$, where

$$
\begin{gathered}
\delta_{n}^{(1)}=\left[r_{n}^{\prime}, \frac{s_{n}^{\prime}\left(s_{n}^{\prime}+1\right)}{2}\right], \quad \delta_{n}^{(2)}=\left[l_{n}^{\prime}, \frac{\left(s_{n}^{\prime}+1\right)\left(s_{n}^{\prime}+2\right)}{2}-1\right], \\
\frac{\left(s_{n}^{\prime}-1\right) s_{n}^{\prime}}{2}<r_{n}^{\prime} \leq \frac{s_{n}^{\prime}\left(s_{n}^{\prime}+1\right)}{2} .
\end{gathered}
$$


Because we have rank $P_{\sigma_{n}^{(1)}}=\operatorname{rank} P_{\sigma_{n}^{(2)}}$ we can find $W \in D_{1}^{\omega} \mathscr{A}$ such that $W^{*} W=P_{\sigma^{(2)}}$ and $W W^{*}=P_{\sigma^{(1)}}$. Let $U \in \alpha(\tilde{W})$. Using Lemma 1.2 and Proposition 3.12 we may suppose that in fact

$$
\tilde{Q}_{\delta^{(1)}}=\alpha\left(\tilde{P}_{\sigma^{(1)}}\right), \quad \tilde{Q}_{\delta^{(2)}}=\alpha\left(\tilde{P}_{\sigma^{(2)}}\right), \quad U \in \mathscr{B}_{(1)}^{\gamma}, \quad Q_{\delta^{(1)}} U=U Q_{\delta^{(2)}}=U .
$$

Applying Lemma 4.2 we know that

$$
\Delta_{1}^{\gamma} U:\left(Q_{\delta^{(2)}} H\right) \rightarrow\left(Q_{\delta^{(1)}} H\right)
$$

is a Fredholm operator, and this obviously implies

$$
\operatorname{rank} Q_{\delta_{n}^{(1)}}=\operatorname{rank} Q_{\delta_{n}^{(2)}}, \quad n \geq n_{0},
$$

for some $n_{0} \in \mathbf{N}$. This means

$$
l_{n}^{\prime}=s_{n}^{\prime}+r_{n}^{\prime}=v\left(r_{n}^{\prime}\right), \quad n \in N,
$$

or equivalently $\hat{\alpha} \hat{v}(\hat{\sigma})=\hat{v} \hat{\alpha}(\hat{\sigma})$, contradicting our working assumptions.

4.5. LeMMA. Let $\omega_{0}$ be as in Lemma 4.3 and assume $\hat{\alpha}^{\omega}\left(\hat{\omega}_{0}\right)=\hat{\omega}_{0}$. Then we have

$$
\alpha\left(\tilde{I}_{\mathscr{A}}^{\omega}\right)=\tilde{I}_{\mathscr{B}}^{\gamma} \quad \text { and } \quad \alpha\left(\tilde{F}_{\mathscr{A}}^{\omega}\right)=\tilde{F}_{\mathscr{B}}^{\gamma}
$$

Proof. Suppose that there exists $A \in I_{\mathscr{A}}^{\omega}, B \in \alpha(\tilde{A})$ with $B \notin I_{\mathscr{B}}^{\gamma}$. Since by Lemma 1.1 we have

$$
\varlimsup_{n \rightarrow \infty}\left\|\left(I-Q^{(\gamma, n-1)}\right) B Q^{(\gamma, v(n)-1)}\right\|>0
$$

we can find $k_{n}, l_{n}, r_{n}, s_{n} \in \mathbf{N}$ such that

$$
\begin{gathered}
s_{n}=v\left(k_{n}\right)-1, \quad k_{n} \leq l_{n}<k_{n+1}, \quad r_{n} \leq s_{n}<r_{n+1}, \\
\left\|Q_{\left[k_{n}, l_{n}\right]}^{\gamma} B Q_{\left[r_{n}, s_{n}\right]}^{\gamma}\right\| \geq a>0 .
\end{gathered}
$$

Let us put $\delta=\bigcup_{n \in \mathrm{N}}\left[k_{n}, l_{n}\right], \delta_{2}=\bigcup_{n \in \mathrm{N}}\left[r_{n}, s_{n}\right]$ and pick $\sigma_{1}, \sigma_{2} \in \mathscr{N}$ such that $\hat{\delta}_{1}=\hat{\alpha}^{\omega}\left(\hat{\sigma}_{1}\right), \hat{\delta}_{2}=\hat{\boldsymbol{\alpha}}^{\omega}\left(\hat{\sigma}_{2}\right)$. Using Lemma 3.4 , Lemma 4.4 and Lemma 1.2 we may assume that we have

$$
\sigma_{1}=\bigcup_{n \in \mathbf{N}}\left[k_{n}^{\prime}, l_{n}^{\prime}\right], \quad \sigma_{2}=\bigcup_{n \in \mathbf{N}}\left[r_{n}^{\prime}, s_{n}^{\prime}\right], \quad \tilde{Q}_{\delta_{1}}^{\gamma}=\alpha\left(\tilde{P}_{\sigma_{1}}^{\omega}\right), \quad \tilde{Q}_{\delta_{2}}^{\gamma}=\alpha^{\omega}\left(\tilde{P}_{\sigma^{2}}\right)
$$

where $l_{n}^{\prime}<k_{n+1}^{\prime}, v\left(k_{n}^{\prime}\right)-1=s_{n}^{\prime}<r_{n+1}^{\prime}$. Applying Lemma 1.5 and decreasing $\sigma_{1}$ and $\sigma_{2}$ if necessary, we finally may assume that

$$
P_{\sigma_{1}}^{\omega} A P_{\sigma_{2}}^{\omega}-\sum_{n \in N} P_{\left[k_{n}^{\prime}, l_{n}^{\prime}\right]}^{\omega} A P_{\left[r_{n}^{\prime}, s_{n}^{\prime}\right]}^{\omega} \in K(H) .
$$

However since $A \in I_{\mathscr{A}}^{\omega}$, we derive

$$
\sum_{n \in N} P_{\left[k_{n}^{\prime}, l_{n}^{\prime}\right]}^{\omega} A P_{\left[r_{n}^{\prime}, s_{n}^{\prime}\right]}^{\omega}=0 \quad \text { and } \quad \tilde{Q}_{\delta_{1}}^{\gamma} \tilde{B} \tilde{Q}_{\delta_{2}}=0
$$


thus contradicting the relation

$$
\left\|Q_{\left[k_{n}, l_{n}\right]} B Q_{\left[r_{n}, s_{n}\right]}\right\| \geq a>0 .
$$

This shows that we have $\alpha\left(\tilde{I}_{\mathscr{A}}^{\omega}\right) \subset \tilde{I}_{\mathscr{B}}^{\gamma}$ and by symmetry $\alpha\left(\tilde{I}_{\mathscr{A}}^{\omega}\right)=\tilde{I}_{\mathscr{B}}^{\gamma}$. The relation $\alpha\left(\tilde{F}_{\mathscr{A}}^{\omega}\right)=\tilde{F}_{\mathscr{B}}^{\omega}$ can be proved analogously, thus completing the proof.

Let $A \in I_{\mathscr{A}}^{\omega}, A^{\prime} \in F_{\mathscr{A}}^{\omega}$. Then we have

$$
P_{n}^{\omega} A P_{k}^{\omega}=0, \quad k \leq v(n)-1, \quad P_{n}^{\omega} A^{\prime} P_{k}^{\omega}=0, \quad k \leq v(n) .
$$

We define

$$
D_{v}^{\omega}: L(H) \rightarrow L(H) \text { and } \Delta_{v}^{\gamma}: L(H) \rightarrow L(H)
$$

by

$$
D_{v}^{\omega} T=\sum_{n \in \mathbf{N}} P_{n}^{\omega} T P_{v(n)}^{\omega}, \quad \Delta_{v}^{\gamma} T=\sum_{n \in N} Q_{n}^{\gamma} T Q_{v(n)}^{\gamma}, \quad T \in L(H) .
$$

We have $A \in F_{\mathscr{A}}^{\omega}$ iff $D_{v}^{\omega} A=0$ or equivalently

$$
I_{\mathscr{A}}^{\omega}=\left(D_{v}^{\omega} \mathscr{A}\right)+F_{\mathscr{A}}^{\omega} \text {. }
$$

4.6. LemMA. Let $\omega_{0}$ be as in Lemma 4.3 and suppose $\hat{\alpha}^{\omega}\left(\hat{\omega}_{0}\right)=\hat{\omega}_{0}$. Let $P, P^{\prime} \in \mathscr{A}$ be selfadjoint projections and let $W \in D_{v}^{\omega} \mathscr{A}$ be such that

$$
P^{\prime} W: P H \rightarrow P^{\prime} H
$$

is a Fredholm operator. Let $Q=Q^{2} \in b, Q^{\prime}=Q^{\prime 2} \in B, \tilde{Q}=\alpha(\tilde{P}), \tilde{Q}^{\prime}=$ $\alpha(\tilde{P}), U \in \alpha(\tilde{W})$ and put

$$
U_{v}=\left(\Delta_{0}^{\gamma} Q^{\prime}\right)\left(\Delta_{v}^{\gamma} U\right):\left(\Delta_{0}^{\gamma} Q\right) H \rightarrow\left(\Delta_{0}^{\gamma} Q^{\prime}\right) H .
$$

Then $U_{v}$ is a Fredholm operator.

Proof. We repeat the proof of Lemma 4.2, using Lemma 4.5 in place of Proposition 3.12.

4.7. Lemma. Let $P=P^{2} \in \mathscr{A}$ be such that $\operatorname{rank} P_{n}^{\omega} P P_{n}^{\omega}=n_{0}$, for $n \in \operatorname{supp}_{\mathscr{Q}^{\omega}}(P)$. Then there exists $Q=Q^{2} \in \mathscr{B}$ such that

$$
\tilde{Q}=\alpha(\tilde{P}), \quad \operatorname{rank} Q_{n}^{\gamma} Q Q_{n}^{\gamma}=n_{0} \quad \text { for } n \in \operatorname{supp}_{\mathscr{B}^{\gamma}}(Q) .
$$

Proof. Pick $Q^{\prime} \in \alpha(\tilde{P})$ and as in the proof of Lemma 2.3 put

$$
\sigma=\left\{n \in \operatorname{supp}_{\mathscr{B} \gamma}\left(Q^{\prime}\right): \operatorname{rank} Q_{n}^{\gamma} Q^{\prime} Q_{n}^{\gamma}>n_{0}\right\}
$$

and

$$
\delta=\left\{n \in \operatorname{supp}_{\mathscr{B}^{\gamma}}\left(Q^{\prime}\right): \operatorname{rank} Q_{n}^{\gamma} Q^{\prime} Q_{n}^{\gamma}<n\right\}
$$


If $\sigma \in \mathscr{N}_{0}$ we can find $T \in \Delta_{0}^{\gamma} \mathscr{B}$ such that

$$
\tilde{T}^{n_{0}+1}=0, \quad \tilde{T}^{n_{0}} \neq 0 \quad \text { and }\left(\Delta_{0}^{\gamma} Q^{\prime}\right) T=T\left(\Delta_{0}^{\gamma} Q^{\prime}\right)=T .
$$

The existence of $T$ can be proved using the freedom we have to use matrix entries involving $\mathscr{B}_{(1)}$. But it is plain that $\tilde{D}_{0}^{\omega} \alpha^{-1}(\tilde{T})$ is a nilpotent of order at most $n$ and because $\left(\tilde{D}_{0}^{\gamma} \alpha^{-1}(\tilde{T})\right)^{n_{0}}=\tilde{D}_{0}^{\gamma} \alpha^{-1}(\tilde{T})^{n_{0}}$, Proposition 3.12 implies $\left(\tilde{D}_{0}^{\gamma} \alpha^{-1}(\tilde{T})\right)^{n_{0}} \neq 0$. The rest of the proof repeats the arguments used in the last part of the proof of Lemma 3.2.

Let us put for each $n \in \mathbf{N}$

$$
\sigma_{n}=\left\{v^{k-1}\left(\frac{n(n+1)}{2}\right)\right\}_{k \in \mathbf{N}}
$$

It is easy to see that we have

$\sigma_{m} \cap \sigma_{n}=\varnothing, \quad m \neq n, \quad \mathbf{N}=\bigcup_{n \in N} \sigma_{n} \quad$ and $\quad v\left(\sigma_{n}\right)=\sigma_{n} \backslash\left\{\frac{n(n+1)}{2}\right\}$.

\subsection{LEMMA. Let us put}

$$
\hat{\mathscr{N}}_{v}=\{\hat{\boldsymbol{\sigma}} \in \hat{\mathscr{N}}: \hat{v}(\hat{\boldsymbol{\sigma}})=\hat{\boldsymbol{\sigma}} \neq \hat{\varnothing}\} .
$$

Then $\hat{\sigma} \in \hat{\mathscr{N}}_{v}$ is minimal in $\hat{\mathscr{N}}_{v}$ if and only if $\hat{\sigma}=\hat{\sigma}_{n}$ for some $n \in N$.

Proof. Let $\sigma^{\prime} \in \hat{\sigma} \in \hat{\mathcal{N}}_{v}$. Since $\mathbf{N}=\bigcup_{n \in \mathbf{N}} \sigma_{n}$ and $v(m)>m, m \in N$ we can find $n \in N$ such that $\sigma^{\prime} \cap \sigma_{n} \notin \mathscr{N}_{0}$. For evey $s \in \sigma^{\prime} \cap \sigma_{n}$ put

$$
g(s)=\sup \left\{k \in \mathbf{N}: v^{m-1}(s) \in \sigma^{\prime} \cap \sigma_{n}, 1 \leq m \leq k\right\}
$$

If the set $\sigma^{\prime \prime}=\left\{s \in \sigma^{\prime} \cap \sigma_{n}: g(s)<\infty\right\}$ is infinite then $v\left(\sigma^{\prime \prime}\right) \cap \sigma^{\prime} \cap \sigma_{n}$ $=\varnothing, \hat{\sigma}^{\prime \prime} \neq \hat{\varnothing}$. But this is a contradiction because $\hat{v}\left(\hat{\sigma}^{\prime} \cap \hat{\sigma}_{n}\right)=\hat{\sigma}^{\prime} \cap \hat{\sigma}_{n}$. It follows that there exists $s \in \sigma^{\prime} \cap \sigma_{n}$ such that $g(s)=\infty$, and this obviously implies

$$
\sigma^{\prime} \cap \sigma_{n} \supset\left\{v^{k-1}(s)\right\}_{k \in \mathbf{N}} \simeq \sigma_{n} \supset \sigma^{\prime} \cap \sigma_{n} .
$$

This shows that any $\hat{\sigma}_{n}$ is minimal in $\hat{\mathscr{N}}_{v}$. If $\hat{\sigma}^{\prime}$ is minimal and $\hat{\sigma}^{\prime} \cap \hat{\sigma}_{n} \neq$ $\hat{\varnothing}$, the above reasoning completes the proof by implying that

$$
\hat{\sigma}^{\prime}=\hat{\sigma}^{\prime} \cap \hat{\sigma}_{n}=\hat{\sigma}_{n} .
$$

Let $R\left(\mathscr{N}_{\mathscr{A}}^{\omega}\right)$ denote the set of all ranks of the block-diagonal projections $\left\{P_{n}^{\omega}\right\}_{n \in \mathrm{N}}$ and analogously we consider $R\left(\mathscr{N}_{\mathscr{B}}^{\gamma}\right)$.

4.9. LEMMA. Let $\omega_{0}$ be as in Lemma 4.3 and assume

$$
\hat{\alpha}^{\omega}\left(\omega_{0}\right)=\hat{\omega}_{0}, \quad \operatorname{rank} P_{n}^{\omega}<\operatorname{rank} P_{n+1}^{\omega}
$$

Then $\overline{R\left(\mathscr{N}_{\mathscr{A}}^{\omega}\right)} \cap \widehat{R\left(\mathscr{N}_{\mathscr{B}}^{\gamma}\right)} \neq \hat{\varnothing}$ 
Proof. Let $P_{n}^{\prime}=P_{n}^{\prime 2}=P^{\prime *} \in \mathscr{A}, P_{n}^{\prime} \leq P_{n}^{\omega}$ be such that

$$
\operatorname{rank} P_{v^{k-1}(n(n+1) / 2)}^{\prime}=\operatorname{rank} P_{n(n+1) / 2}^{\omega}, \quad k, n \in N,
$$

and put $P^{\prime}=\sum_{n \in \mathbf{N}} P_{n}^{\prime}$. Using Lemma 1.2, Lemma 1.3 and Lemma 4.4 we may assume that we have $\tilde{Q}^{\prime}=\alpha\left(\tilde{P}^{\prime}\right), \quad \tilde{Q}_{v(N)}^{\prime}=\alpha\left(P_{v(N)}^{\prime}\right), \quad \tilde{Q}_{v(N)}^{\gamma}=$ $\alpha\left(\tilde{P}_{v(N)}^{\omega}\right)$, where

$$
Q^{\prime}=Q^{\prime 2}=Q^{*} \in \mathscr{B}, \quad Q^{\prime}=\sum_{n \in \mathbf{N}} Q_{n}^{\prime}, Q_{n}^{\prime} \leq Q_{n}^{\gamma} .
$$

Because $\hat{\alpha}^{\omega}\left(\hat{\omega}_{0}\right)=\hat{\omega}_{0}$ we can take $Q_{n(n+1) / 2}^{\prime}=Q_{n(n+1) / 2}^{\gamma}, n \in \mathbf{N}$. Let $W \in \mathscr{A}$ be a partial isometry such that

$$
P_{m}^{\omega} W P_{v(n)}^{\omega}=0 \quad \text { whenever } m \neq n \quad \text { and } \quad W W^{*}=P^{\prime}, \quad W^{*} W=P_{v(N)}^{\prime} .
$$

Let $U \in \mathscr{B}$ be such that $\tilde{U}=\alpha(\tilde{W})$ and put

$$
U_{v}=Q^{\prime}\left(\Delta_{v}^{\gamma} U\right):\left(Q_{v(N)}^{\prime} H\right) \rightarrow\left(Q^{\prime} H\right) .
$$

Since we have $\hat{\alpha}^{\omega} \hat{v}(\hat{N})=\hat{v} \hat{\alpha}^{\omega}(\hat{N})=\hat{v}(\hat{N})$ (see Lemma 4.4) applying Lemma 4.6 we know that $U_{v}$ is Fredholm and this implies

$$
\operatorname{rank}\left(Q_{v^{k-1}(n)}^{\prime} U Q_{v^{k}(n)}^{\prime}\right)=d_{n}, \quad k \in \mathbf{N}, n \geq n_{0}
$$

for some $n_{0} \in \mathbf{N}$. Choose $r=(m(m+1) / 2) \geq n_{0}$ and $\sigma \in \mathcal{N}$ such that $\hat{\boldsymbol{\alpha}}^{\omega}(\hat{\boldsymbol{\sigma}})=\hat{\boldsymbol{\sigma}}_{m}$. It is plain that $\hat{\boldsymbol{\sigma}}$ is minimal in $\hat{N}_{v}$, thus by Lemma 4.8 , we derive $\hat{\sigma}=\hat{\sigma}_{l}$ for some $l \in \mathbf{N}$. Because by our hypothesis we have

$$
\operatorname{rank} P_{v^{k-1}(l(l+1) / 2)}^{\prime}=\operatorname{rank} P_{l(l+1) / 2}^{\omega}, \quad k \in \mathbf{N},
$$

applying Lemma 4.7 we get $\operatorname{rank} Q_{r}^{\gamma}=\operatorname{rank} P_{l(l+1) / 2}^{\omega}$, for infinitely many $r$ 's which completes the proof.

The map $\hat{\alpha}^{\omega}$ depends on both $\omega$ and $\gamma$. The dependence on $\gamma$, when $\omega$ is fixed is an indexation matter. The next lemma will allow us to optimize the choice of $\gamma$ (modulo $\simeq$ ), with respect to $\omega_{0}$.

4.10. LEMMA. We can choose $\gamma \in \hat{\alpha}(\hat{\omega})$ such that $\hat{\alpha}^{\omega}\left(\hat{\omega}_{0}\right)=\hat{\omega}_{0}$.

Proof. Let $\gamma^{\prime} \in \hat{\alpha}(\hat{\omega})$ be of the form $\gamma^{\prime}=\left\{\alpha_{n}^{\prime}\right\}_{n \in \mathbf{N}}, \alpha_{n}^{\prime}<\alpha_{n+1}^{\prime}$. By Lemma 4.3 we know that we have $\hat{\alpha}_{l}^{\omega}\left(\hat{\omega}_{0}\right)=\hat{\tau}^{m^{\prime}}\left(\hat{\omega}_{0}\right)$ for some integer $m^{\prime}$. If we take $\gamma=\left\{\alpha_{n}\right\}_{n \in \mathbf{N}}$, where $\alpha_{n}=\alpha_{n+m^{\prime}}^{\prime}$ for large $n$ and $\delta \in \hat{\tau}^{m^{\prime}}\left(\hat{\omega}_{0}\right)$, then using Lemma 3.1 we deduce $\hat{\alpha}\left(\hat{\omega}_{\omega_{0}}\right)=\hat{\gamma}_{\delta}^{\prime}=\hat{\gamma}_{\omega_{0}}$ or equivalently $\hat{\boldsymbol{\alpha}}^{\omega}\left(\hat{\omega}_{0}\right)=\hat{\omega}_{0}$. 
4.11. Lemma. Suppose that $\operatorname{rank} P_{n}^{\omega}<\operatorname{rank} P_{n+1}^{\omega}, n \in \mathbf{N}$. Then there exist an integer $m_{0}$ and $n_{0} \in \mathbf{N}$ such that

$$
\operatorname{rank} P_{n}^{\omega}=\operatorname{rank} Q_{n+m_{0}}^{\gamma} \text { and } n \geq n_{0}, \quad \hat{\alpha}^{\omega}=\hat{\tau}^{m_{0}} .
$$

Proof. Arguing as in Lemma 4.4 we may suppose rank $Q_{n}^{\gamma}<$ $\operatorname{rank} Q_{n+1}^{\gamma}, n \in \mathbf{N}$. Then the first relation in the statement is obviously equivalent to the relation $R\left(\mathscr{N}_{\mathscr{A}}^{\omega}\right) \simeq R\left(\mathcal{N}_{\mathscr{B}}^{\gamma}\right)$. Suppose $R\left(\mathscr{N}_{\mathscr{Q}}^{\omega}\right) \neq R\left(\mathscr{N}_{\mathscr{B}}^{\gamma}\right)$. Then replacing $\mathscr{A}$ and $\mathscr{B}$ by suitable compression algebras (see the preliminaries to Lemma 3.1) we reduce to the case $R\left(\mathscr{N}_{\mathscr{A}}^{\omega}\right) \cap R\left(\mathscr{N}_{\mathscr{B}}^{\gamma}\right)=$ $\varnothing$. But because $\widehat{R\left(\mathcal{N}_{\mathscr{B}}^{\gamma}\right)}$ does not depend on $\hat{\gamma}$, applying Lemma 4.10 we may suppose $\hat{\alpha}^{\omega}\left(\hat{\omega}_{0}\right)=\hat{\omega}_{0}$ and Lemma 4.9 implies $R\left(\overline{\mathscr{N}_{\mathscr{A}}^{\omega}}\right) \cap \overline{R\left(\mathcal{N}_{\mathscr{B}}^{\gamma}\right)} \neq$ $\hat{\varnothing}$, a contradiction. Thus we conclude that we have

$$
\operatorname{rank} P_{n}^{\omega}=\operatorname{rank} Q_{n+m_{0}}^{\gamma}, \quad n \geq n_{0} .
$$

Let $\sigma \in \mathscr{N}, \delta \in \alpha^{\omega}(\hat{\sigma})$. Applying the first part of the proof to the compression algebras $A_{\mathscr{P}_{\sigma}^{\omega}}$ and $B_{Q_{\delta}^{\gamma}}$ we deduce

$$
\left\{\operatorname{rank} P_{n}^{\omega}: n \in \sigma\right\} \simeq\left\{\operatorname{rank} Q_{n}^{\gamma}: n \in \delta\right\}
$$

and this obviously implies $\hat{\delta}=\hat{\tau}^{m_{0}}(\hat{\sigma})$.

4.12. Corollary. Let $\sigma=\bigcup_{n \in \mathrm{N}}\left[b_{n}, c_{n}\right], b_{n} \leq c_{n}<b_{n+1}$ and let $\delta \in$ $\hat{\alpha}(\hat{\sigma})$ where $\delta=\bigcup_{n \in \mathrm{N}}\left[b_{n}^{\prime}, c_{n}^{\prime}\right], \quad b_{n}^{\prime} \leq c_{n}^{\prime}<b_{n+1}^{\prime}$. If $\operatorname{rank} P_{\left[b_{n}, c_{n}\right]}<$ rank $P_{\left[b_{n+1}, c_{n+1}\right]}$ then there exists an integer $m_{0}$ and $n_{0} \in \mathbf{N}$ such that

$$
\operatorname{rank} P_{\left[b_{n}, c_{n}\right]}=\operatorname{rank} Q_{\left[b_{n+m_{0}}^{\prime}, c_{n+m_{0}}^{\prime}\right]}, \quad n \geq n_{0} .
$$

Proof. We reduce to the application of Lemma 4.1 to the compression algebras $\mathscr{A}_{\mathscr{P}_{\sigma}}$ and $\mathscr{B}_{Q_{\delta}}$.

4.13. Lemma. Suppose that there exists an integer $m_{0}$ such that $\hat{\alpha}=\hat{\tau}^{m_{0}}$. Then there exists $n_{0} \in \mathbf{N}$ such that

$$
\text { rank } P_{n}=\operatorname{rank} Q_{n+m_{0}}, \quad n \geq n_{0} .
$$

Proof. Suppose the contrary. Then we can reduce to the case

$$
\operatorname{rank} P_{a_{n}} \neq \operatorname{rank} Q_{\alpha_{n}}, \quad \operatorname{rank} P_{n}^{\omega}<\operatorname{rank} P_{\left(a_{n}, a_{n+1}\right)}, \quad n \in \mathbf{N},
$$

where $\alpha_{n}=a_{n}+m_{0}$ for large $n$. Applying Lemma 4.11 we can find an integer $m_{0}^{\prime}$ such that

$$
\operatorname{rank} P_{n}^{\omega}=\operatorname{rank} Q_{n+m_{0}^{\prime}}^{\gamma}, \quad n \geq n_{0}, \quad \hat{\alpha}^{\omega}=\hat{\tau}^{m_{0}^{\prime}} .
$$


Using Lemma 3.11 we infer $\hat{\alpha}\left(\hat{\omega}_{\sigma}\right)=\hat{\tau}^{m_{0}}\left(\hat{\omega}_{\sigma}\right)=\hat{\gamma}_{\delta}$ for every $\sigma \in \mathscr{N}$, $\delta \in \hat{\tau}^{m_{0}^{\prime}}(\hat{\sigma})$ which yields

$$
\left\{a_{n}+m_{0}\right\}_{n \in \sigma} \simeq\left\{a_{n+m_{0}^{\prime}}+m_{0}\right\}_{n \in \sigma} .
$$

This obviously implies $m_{0}^{\prime}=0$ and in particular

$$
\operatorname{rank} P_{n}^{\omega}=\operatorname{rank} Q_{n}^{\gamma}, \quad n \geq n_{0}, \quad \hat{\alpha}^{\omega}(\hat{\sigma})=\hat{\sigma}, \quad \sigma \in \mathscr{N} .
$$

Applying Corollary 4.12 we can find an integer $m_{0}^{\prime \prime}$ such that

$$
\text { rank } P_{\left[a_{n-1}+1, a_{n}-1\right]}=\operatorname{rank} Q_{\left[\alpha_{n-1+m^{\prime}}+1, \alpha_{n+m^{\prime}}-1\right]}, \quad n \geq n_{0} .
$$

Using Lemma 3.11 and Lemma 3.13 we deduce as before $m_{0}^{\prime \prime}=0$ and

$$
\text { rank } P_{\left[a_{n-1}+1, a_{n}-1\right]}=\operatorname{rank} Q_{\left[\alpha_{n-1}+1, \alpha_{n}-1\right]}, \quad n \geq n_{0} .
$$

Subtracting (2) from (1) we get rank $P_{a_{n}}=\operatorname{rank} Q_{\alpha_{n}}, n \geq n_{0}$, a contradiction.

The Proof of Theorem 4.1. Using Lemma 4.13 it suffices to prove that we have $\hat{\alpha}=\hat{\tau}^{m_{0}}$ for some integer. Applying Lemma 3.1 we shall suppose rank $P_{n}=\operatorname{rank} Q_{n}=1, n \in \mathbf{N}$. Take $\omega=\omega_{0}, m_{0}$ produced by Lemma 4.3 such that $\hat{\alpha}(\hat{\omega})=\hat{\tau}^{m_{0}}(\hat{\omega})$ and $\alpha_{n}=a_{n}+m_{0}$ for large $n$. Suppose that we have $\hat{\alpha}\left(\hat{\sigma}^{\prime}\right) \neq \hat{\tau}^{m_{0}}\left(\sigma^{\prime}\right)$ for some $\sigma^{\prime} \in \mathscr{N}$ and pick $\delta^{\prime} \in \hat{\alpha}\left(\hat{\sigma}^{\prime}\right)$. Let $\sigma_{0}^{\prime} \in \mathscr{N}$ be such that $\sigma^{\prime}=\left\{c_{n}\right\}_{m \in \sigma_{0}^{\prime}}, \delta^{\prime}=\left\{c_{n}^{\prime}\right\}_{n \in \sigma_{0}^{\prime}}$ and

$$
a_{n-1}<c_{n} \leq a_{n}, \quad \alpha_{n-1}<c_{n}^{\prime} \leq \alpha_{n}, \quad n \in \sigma_{0}^{\prime}
$$

Case I. The sequence $\left\{c_{n}-a_{n-1}\right\}_{n \in \sigma_{0}^{\prime}}$ is strictly increasing. Let us put

$$
P=\sum_{n \in \sigma_{0}^{\prime}} P_{\left[a_{n-1}, c_{n}\right]}, \quad Q=\sum_{n \in \sigma_{0}^{\prime}} Q_{\left[\alpha_{n-1}, c_{n}^{\prime}\right]}
$$

and assume (after a similarity) that $\tilde{Q}=\hat{\alpha}(\tilde{P})$. Decreasing $\sigma^{\prime}$ if necessary we may assume $\hat{\delta}^{\prime} \cap \hat{\tau}^{m_{0}}\left(\hat{\sigma}^{\prime}\right)=\hat{\varnothing}$ and then (because $\alpha_{n}=a_{n}+m_{0}$, $\left.c_{n}^{\prime} \neq c_{n}+m_{0}, n \in \sigma_{0}^{\prime}\right)$

$$
\left\{\operatorname{rank} P_{\left[a_{n-1}, c_{n}\right]}\right\}_{n \in \sigma_{0}^{\prime}} \cap\left\{\operatorname{rank} Q_{\left[\alpha_{n-1}, c_{n}^{\prime}\right]}\right\}_{n \in \sigma_{0}^{\prime}}=\varnothing .
$$

But making a natural identification and applying Lemma 4.11 to

$$
\alpha_{P}: \tilde{\mathscr{A}}_{P} \rightarrow \tilde{\mathscr{B}}_{Q}
$$

we deduce

$$
\left\{\operatorname{rank} P_{\left[a_{n-1}, c_{n}\right]}\right\}_{n \in \sigma_{0}^{\prime}} \simeq\left\{\operatorname{rank} Q_{\left[\alpha_{n-1}, c_{n}^{\prime}\right]}\right\}_{n \in \sigma_{0}^{\prime}}
$$

and this is a contradiction. 
Case II. The sequence $\left\{c_{n}-a_{n-1}\right\}_{n \in \sigma_{0}^{\prime}}$ is not strictly increasing. If $\left\{c_{n}-a_{n-1}\right\}_{n \in \sigma_{0}^{\prime}}$ is unbounded, decreasing $\sigma^{\prime}$ we can reduce to Case I. If $\left\{c_{n}-a_{n-1}\right\}_{n \in \sigma_{0}^{\prime}}$ is bounded, then decreasing $\sigma^{\prime}$ we may suppose that the sequence $\left\{a_{n}-c_{n}\right\}_{n \in \sigma_{0}^{\prime}}$ is strictly increasing and then proceed as before.

REMARK. The best we could expect would be that $\alpha$ is implemented by a Calkin similarity. Though Theorem 4.1 does not provide such a similarity, it suggests that its existence is a reasonable conjecture. It also seems reasonable to conjecture that the conclusion of Theorem 4.1 holds true if we assume only that the algebras $\tilde{\mathscr{A}}_{(0)}$ and $\tilde{\mathscr{B}}_{(0)}$ are isomorphic.

\section{REFERENCES}

[1] W. Arveson, Ten lectures on operator algebras, CBMS Regional Conference, Lubbock, Texas, 1983.

[2] J. Erdos, Non-selfadjoint operator algebras, Proc. Royal Irish Acad., 81A (1981), 127-145.

[3] T. Fall, W. Arveson and P. Muhly, Perturbations of nest algebras, J. Operator Theory, 1 (1979), 135-150.

[4] J. Plastiras, Quasitriangular operator algebras, Pacific J. Math., 64 (1976), 543-550.

[5] _ Compact perturbations of von Neumann algebras, Trans. Amer. Math. Soc., 234 (1977), 561-577.

[6] J. R. Ringrose, On some algebras of operators, Proc. London Math. Soc., 15 (1965), 61-83.

Received January 28, 1985.

Arizona State University

TEMPE, AZ 85287

AND

UNIVERSITY OF NEBRASKA

LINCOLN, NE 68588 


\section{PACIFIC JOURNAL OF MATHEMATICS EDITORS}

\author{
V. S. VARAdarajan (Managing Editor) \\ University of California \\ Los Angeles, CA 90024 \\ Hebert Clemens \\ University of Utah \\ Salt Lake City, UT 84112 \\ Charles R. DePrima \\ California Institute of Technology \\ Pasadena, CA 91125
}

R. FINN

Stanford University

Stanford, CA 94305

HeRManN FLASChKa

University of Arizona

Tucson, AZ 85721

RAMESH A. GANGOlli

University of Washington

Seattle, WA 98195

ROBION KIRBY

University of California

Berkeley, CA 94720

\author{
C. C. MOORE \\ University of California \\ Berkeley, CA 94720 \\ H. SAMELSON \\ Stanford University \\ Stanford, CA 94305 \\ HAROLD STARK \\ University of California, San Diego \\ La Jolla, CA 92093
}

\section{ASSOCIATE EDITORS}

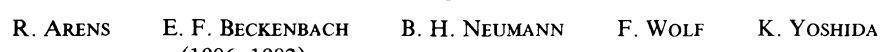

(1906-1982)

\section{SUPPORTING INSTITUTIONS}

\begin{abstract}
UNIVERSITY OF ARIZONA
UNIVERSITY OF BRITISH COLUMBIA

CALIFORNIA INSTITUTE OF TECHNOLOGY

UNIVERSITY OF CALIFORNIA

MONTANA STATE UNIVERSITY

UNIVERSITY OF NEVADA, RENO

NEW MEXICO STATE UNIVERSITY

OREGON STATE UNIVERSITY
\end{abstract}

\author{
UNIVERSITY OF OREGON \\ UNIVERSITY OF SOUTHERN CALIFORNIA \\ STANFORD UNIVERSITY \\ UNIVERSITY OF HAWAII \\ UNIVERSITY OF TOKYO \\ UNIVERSITY OF UTAH \\ WASHINGTON STATE UNIVERSITY \\ UNIVERSITY OF WASHINGTON
}

The Supporting Institutions listed above contribute to the cost of publication of this Journal, but they are not owners or publishers and have no responsibility for its content or policies.

Mathematical papers intended for publication in the Pacific Journal of Mathematics should be in typed form or offset-reproduced (not dittoed), double spaced with large margins. Please do not use built up fractions in the text of the manuscript. However, you may use them in the displayed equations. Underline Greek letters in red, German in green, and script in blue. The first paragraph must be capable of being used separately as a synopsis of the entire paper. In particular it should contain no bibliographic references. Please propose a heading for the odd numbered pages of less than 35 characters. Manuscripts, in triplicate, may be sent to any one of the editors. Please classify according to the scheme of Math. Reviews, Index to Vol. 39. Supply name and address of author to whom proofs should be sent. All other communications should be addressed to the managing editor, or Elaine Barth, University of California, Los Angeles, California 90024.

There are page-charges associated with articles appearing in the Pacific Journal of Mathematics. These charges are expected to be paid by the author's University, Government Agency or Company. If the author or authors do not have access to such Institutional support these charges are waived. Single authors will receive 50 free reprints; joint authors will receive a total of 100 free reprints. Additional copies may be obtained at cost in multiples of 50 .

The Pacific Journal of Mathematics is issued monthly as of January 1966. Regular subscription rate: $\$ 190.00$ a year (5 Vols., 10 issues). Special rate: $\$ 95.00$ a year to individual members of supporting institutions.

Subscriptions, orders for numbers issued in the last three calendar years, and changes of address should be sent to Pacific Journal of Mathematics, P.O. Box 969, Carmel Valley, CA 93924, U.S.A. Old back numbers obtainable from Kraus Periodicals Co., Route 100, Millwood, NY 10546.

The Pacific Journal of Mathematics at P.O. Box 969, Carmel Valley, CA 93924 (ISSN 0030-8730) publishes 5 volumes per year. Application to mail at Second-class postage rates is pending at Carmel Valley, California, and additional mailing offices. Postmaster: Send address changes to Pacific Journal of Mathematics, P.O. Box 969, Carmel Valley, CA 93924.

PUBLISHED BY PACIFIC JOURNAL OF MATHEMATICS, A NON-PROFIT CORPORATION

Copyright $\odot 1986$ by Pacific Journal of Mathematics 


\section{Pacific Journal of Mathematics}

\section{Vol. 122, No. 2 \\ February, 1986}

Gideon Amit and David Chillag, On a question of Feit concerning character values of finite solvable groups ......................257

Constantin Gelu Apostol and Frank Larkin Gilfeather, Isomorphisms modulo the compact operators of nest algebras ................263

Parviz Azimi and James Neil Hagler, Examples of hereditarily $l^{1}$ Banach

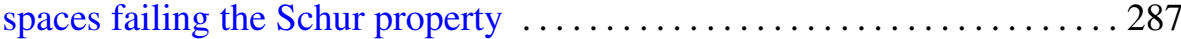

Brian Evan Blank, Boundary behavior of limits of discrete series representations of real rank one semisimple groups . . . . . . . . . . 299

Jeffrey Carroll, Some undecidability results for lattices in recursion theory

Gerald Howard Cliff and Alfred Rheinhold Weiss, Crossed product and

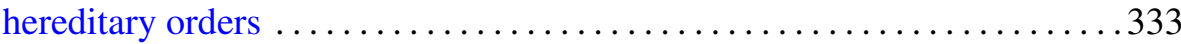

Ralph Cohen, Realizing transfer maps for ramified coverings . . . . . . . . 347

Ronald James Evans, Hermite character sums . .................. 357

C. L. Frenzen and Roderick Sue-Chuen Wong, Asymptotic expansions of the Lebesgue constants for Jacobi series . . . . . . . . . . . . . . . . 391

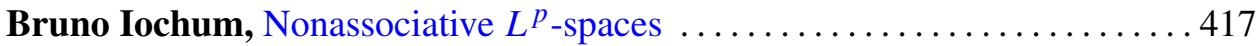

John McDonald, Unimodular approximation in function algebras ....... 435

John Robert Quine, Jr., Ramification and unintegrated value distribution . . 441

Marc Raphael, Commutants of quasisimilar subnormal operators ........ 449

Parameswaran Sankaran and Peter Zvengrowski, On stable

parallelizability of flag manifolds

Helga Schirmer, A relative Nielsen number

Barry Simon, Schrödinger semigroups on the scale of Sobolev spaces . . . . . 475

Viakalathur Shankar Sunder, Stochastic integration in Fock space

Jan de Vries, A note on the $G$-space version of Glicksberg's theorem 